\title{
A computational framework for immiscible three phase flow in deformable porous media
}

\author{
Alessandro Gajo ${ }^{\mathrm{a}}$, Francesco Cecinato ${ }^{\mathrm{a}, *}$, Benjamin Loret $^{\mathrm{b}}$ \\ ${ }^{a}$ Università di Trento, DICAM, Via Mesiano 77, 38123 Trento, Italy \\ ${ }^{\mathrm{b}}$ Université de Grenoble, Laboratoire 3SR, 38041 Grenoble Cedex 9, France
}

\section{A R T I C L E I N F O}

\section{Keywords:}

Immiscible fluids

Capillary pressures

Relative permeabilities

Gas injection

Imbibition

Finite element

Time marching scheme

\begin{abstract}
A B S T R A C T
Several soil decontamination processes and enhanced oil recovery techniques involve the co-existence of three immiscible fluids, such as water, a nonaqueous phase liquid and a gas. In this work, a computational framework based on the individual mass balance of each phase is developed, aimed at simulating three-phase flow in a deformable rock through the finite element method, without resorting to specific simplifications that are usually required by standard numerical schemes. Key ingredients of the model are: expression of the residual in terms of mass contents, consistent lumping of the storage terms in the residual and algorithmic (tangent) matrix, consistent integration rules, the use of a minimum relative permeability and a time marching scheme based on trapezoidal integration. Special convective boundary conditions are adopted for pressures to be consistent with the assumed rock wettability properties during co-current imbibition. The resulting numerical scheme can deal with arbitrary saturation and/or pressure boundary conditions. The model is tested by simulating gas injection tests, and both co- and counter-current water imbibition tests, in a deformable core. To assess the performance and robustness of the whole framework, sensitivity analyses are performed upon varying key constitutive, loading and numerical parameters.
\end{abstract}

\section{Introduction}

A number of processes in civil, environmental and hydrocarbon engineering involve the co-existence of three non miscible fluids, typically water, a nonaqueous phase liquid (NAPL, e.g. chlorinated solvent, oil, supercritical $\mathrm{CO}^{2}$ ) and a gas (e.g. air, methane, etc.). Such situations arise for example in groundwater contamination problems (e.g. (Mercer and Cohen, 1990), (Bass et al., 2000)), in enhanced oil recovery techniques such as water alternating gas injection (WAG) or steam assisted gravity drainage (SAGD, e.g. (Butler, 1994)), and in $\mathrm{CO}^{2}$ sequestration associated with coal-bed methane recovery (Plug et al., 2008).

The flow of three immiscible fluids in an undeformable porous medium has long been investigated (e.g., see (Bowen, 1976) for an overview of this topic). The separate mass balance equations for each fluid in a three-fluid system can be found for instance in (Peaceman, 1977). Three-phase flow models are usually obtained building up on two-phase models, for which two alternative approaches have been typically proposed. The first approach considers the individual mass balance of each phase, whereas the second one is based on a manipulation of the mass balance equations, to obtain a global fluid pressure and a total flux. The flux of each phase is then deduced from the total flux through a fractional flow function. While the former approach is typical of hydrology and of unsaturated soil mechanics, the latter approach was introduced in petroleum engineering. The two approaches were first compared by Morel-Seytoux (Morel-Seytoux, 1973), and more recently by Binning and Celia (1999), who investigated their numerical efficiency.

It is worth adding tha, $\mathrm{t}$ in the fractional flow approach for two-phase flow, the governing equations are rewritten in terms of a global pressure and saturation ((Chavent and Jaffré, 1978), (Chen and Ewing, 1997)). Reportedly, a characteristic feature of this approach is that under certain conditions the pressure equation is elliptic while the saturation equation is hyperbolic. Thus, they may be solved through a staggered approach, using for instance mixed FE methods (for the pressure equation) and Lagrangian algorithms (for the saturation equation), and implementing either fully implicit or semi-implicit (e.g. IMPES) schemes.

\footnotetext{
* Corresponding author.

Email address: francesco.cecinato@unitn.it (F. Cecinato)
} 
The fractional flow approach does not however appear directly applicable to the analysis of deforming porous media; in fact the solution provides the global pressure and not the single pressures of each fluid (e.g. see (Yang et al., 2014) for a recent two-phase application). This feature could explain the scarce use of this approach in the field of geomechanics. Moreover, the direct extension of the fractional flow approach from two to three phases is not straightforward (Chavent and Jaffré, 1978). In fact, rewriting the governing equations in terms of a global pressure, a total flux and two saturations generally requires the satisfaction of a total differential condition ((Chavent and Jaffré, 1978), (Chen and Ewing, 1997)) which is not usually satisfied by existing relative permeability and capillary pressure curves (Chen and Ewing, 1997). Chen \& Ewing (Chen and Ewing, 1997) considered different fractional flow formulations, some of which not requiring the satisfaction of a differential condition, although the resulting equations may be strongly coupled. More recent numerical studies adopting fractional flow include (Chen, 2000), (Mosharaf Dehkordi et al., 2014) and (Amiri et al., 2013). In most cases, however, adopting the fractional flow approach, the water-oil capillary pressure $p_{\mathrm{o}}-p_{\mathrm{w}}$ is expressed as a function of the sole water saturation whereas the oil-gas capillary pressure $p_{\mathrm{g}}-p_{\mathrm{o}}$ is expressed as a function of the sole gas saturation. This type of simplifying assumption (after Leverett \& Lewis (Leverett and Lewis, 1941)) brings about limitations in the relative permeability versus saturation relationships.

Compared to fractional flow, approaches based on the individual mass balance have been much less investigated for three-fluid phase problems. Some of the main existing individual mass balance based methods are briefly discussed hereafter. One of the first applications along these lines is due to Faust (1985): neglecting gas pressure gradients and solid skeleton deformability, he reduced the original problem to just two mass balance equations, which were solved with a finite difference scheme with fluid pressures as the primary variables. Such approach is clearly not appropriate to address problems of gas (ie. air or steam) injection and problems of counter-current imbibition (see (Gajo et al., 2017)). Later, Forsyth \& Shao (Forsyth and Shao, 1991) used a control-volume FE approach with upstream weighting, considering three immiscible fluid phases, adopting the simplifying assumption of Leverett \& Lewis (Leverett and Lewis, 1941), and neglecting the solid skeleton deformability. Although the selected primary variables were not clearly stated, a careful treatment of phase appearance/disappearance was later proposed (Forsyth, 1994). More recently, Pao et al. (2001) proposed a three-phase FE formulation with deformable porous medium. However, no detailed development is provided for the constitutive equations relating the saturations to capillary pressures and to relative permeabilities in a three-phase context, and actually only two-phase examples were proposed. Further (Yin et al., 2009), developed a non isothermal FE model for three-phase flow in deformable porous media, resorting to SGS/GSGS method ((Harari, 2004), (Hauke et al., 2004), (Harari and Hauke, 2007)) to avoid spurious oscillations. Unfortunately, validation of this method is provided only for two-phase flow. Finally (Mroginski et al., 2010), presented a FE, three-phase flow model for deformable porous media, which is however based on strongly simplified capillary pressure relationships.

It is worth emphasizing that the major numerical difficulties posed by the above cited approaches arise with the propagation of sharp fronts. If the primary unknowns defining the state of the fluids are the pressures, the field equations are parabolic. Still this statement implies some monotonicity property for the capillary pressures, which is satisfied by the adopted constitutive capillary pressures. The above observations do not provide information on saturations. In fact, transforming the balance equations of mass of the fluids along the lines of the fractional flow technique, the field equations for the saturations turn out to be parabolic convection-diffusion equations. The corresponding plots show propagating fronts (associated with the convective terms) which are somehow smoothed out by the diffusive components (emanating from the capillary pressures). If the capillary pressures were vanishing (as per the Buckley-Leverett problem), the equations for the saturations would be hyperbolic (at least when using the Brooks-Corey relative permeabilities) and the propagating fronts would be expected to be sharp.

To avoid spurious oscillations, different techniques have been proposed, such as diagonalization of the storage matrix ((Neuman, 1973), (Mercer and Faust, 1976), (Celia et al., 1990)), use of upstream weighting on the relative permeability (mostly used in finite difference schemes and extendible to FE with difficulty), addition of a dissipation term to the partial differential equations (Spivak et al., 1977) and, more recently, implementation of an advective-diffusive-reactive differential operator and its adjoint (SGS/GSGS method).

In this work, an attempt is made for the first time (to the best of authors' knowledge) to develop an exhaustive computational framework based on the individual mass balance approach, aimed at simulating three-phase flow in a deformable rock through the FE method, without resorting to specific simplifications that are usually required by existing numerical schemes. The solid displacement vector and the three fluid pressures are used as primary variables. The capillary pressure constitutive expressions proposed by (Gajo et al., 2017) are implemented, although the proposed method is not limited to such expressions, provided some smoothness requirements of the constitutive equations are met, because numerical inversion of the capillary pressure expressions plays a key role in the numerical procedure. The key ingredients of the model are: a) the absence of any linearization in the expression of the residual (namely, the use of a mixed formulation), b) consistent lumping of the storage terms in the residual and the algorithmic (tangent) matrix (to avoid spurious oscillations and to ensure exact mass balance of the three fluids), c) consistent integration rules for the residual and tangent matrix, d) the use of a minimum, vanishingly small (but not null) relative permeability, e) a time marching scheme based on a trapezoidal integration. The iterative scheme is driven by a fully implicit Newton's matrix associated with a line search algorithm. Storage of the algorithmic matrices and equation solving using Crout's method takes advantage of the symmetric skyline profile of the non symmetric Newton's matrix.

One of the main aims of this work is to demonstrate the feasibility of solving rigorously a practical engineering problem involving the flux of three immiscible fluids in a deformable porous medium, using a mixed formulation of the mass balance equations without simplifications dictated by the computational scheme or linearization. In fact, even for the simpler case of two immiscible fluid flow, it is widely accepted that the nonlinear dependency of the degree of saturation on the difference of fluid pressures makes the numerical solution of Richards' equation cumbersome, thus requiring sophisticated numerical methods to overcome convergence problems and/or poor computational efficiency (e.g. see (Casulli and Zanolli, 2010)). The numerical problems associated with three-fluid flow are far more severe compared to two-fluid flow, and have not been fully explored yet. The method proposed here is based on a general capillary pressure relationship, and explores the main sources of numerical difficulties. Further, the numerical expedients implemented in the tangent matrix are described and numerical efficiency is explored. Modifications to the consistent Newton's scheme are especially needed to overcome the computational difficulties occurring when the saturation front approaches the outlet boundary, where severe pressure gradients develop.

Finally, an innovative convective boundary condition with a penalty formulation is proposed for the particular treatment of conditions existing in three-fluid problems. In fact, in this kind of immiscible flow, the boundary condition can be easily switched from a Dirichlet to a Neumann condition and vice versa for one of the fluids, depending 
on the wettability properties of the rock. The resulting numerical scheme can a priori address arbitrary pressure-controlled, saturation-controlled or mixed loading scenarios.

The computational framework is then tested by simulating rapid gas injection tests in an elastic core initially saturated by water and oil, and both co- and counter-current water imbibition tests in an elastic core initially saturated by all three fluids. As regards to wettability, emphasis is laid on water wet rocks (where water is more wetting than oil, which is more wetting than gas), thus the simulations presented in this work are more relevant to sandstones and silicate rocks, rather than carbonates (see (Gajo et al., 2017)). To assess the flow and mechanical implications of the constitutive structure and boundary conditions, and to test the stability and robustness of the whole framework, sensitivity analyses are performed upon varying key constitutive, loading and numerical parameters.

Although the examples provided in this paper apply to the flow of three immiscible fluids, with direct applications in hydrocarbon engineering and groundwater decontamination problems, the approach presented here is fully compatible with the unsaturated soil mechanics framework, such as that laid out by (Khalili and Khabbaz, 1995) and (Loret and Khalili, 2000), involving the flow of two immiscible fluids.

The paper is structured as follows. An overview of the adopted constitutive equations for a porous medium saturated by three fluids is provided in Sect. 2 . The mass balance equations, the constitutive equations of the solids and of fluid transport are outlined in Sect. 3, and the discretized equations are described in Sect. 4. Sect. 5 shows gas injection simulations, Sect. 6 discusses some computational aspects concerning gas injection simulations, while in Sect. 7 imbibition test results are presented. Finally, a short critical overview of the proposed approach is presented in Sect. 8 together with prospective applications.

\section{Constitutive equations for a three-fluid phase porous medium}

\subsection{Relative permeabilities for a three-fluid phase porous medium}

The fluid mixture $\mathscr{K}_{f}=\{\mathrm{w}, \mathrm{o}, \mathrm{g}\}$ is composed of three immiscible fluids, namely water w, oil o and gas g. With $V_{i}$ the volume occupied by the fluid $i$ and $V_{f}$ the total fluid volume, we first consider cartesian coordinates where the axes are the three phase saturations $S_{i}=V_{i} / V_{f} \in[0,1]$ $, i=\mathrm{w}, \mathrm{o}, \mathrm{g}$, satisfying the additional constraint $S_{\mathrm{w}}+S_{\mathrm{o}}+S_{\mathrm{g}}=1$. In the cartesian axes $\left(S_{\mathrm{W}}, S_{\mathrm{O}}, S_{\mathrm{g}}\right)$, the saturations belong to a planar triangular domain $\mathscr{T}=$ WOG defined by the vertices $\mathbf{W}, \mathbf{O}$ and $\mathbf{G}$ respectively corresponding to $100 \%$ water, oil and gas. In fact, the actual saturations range in the smaller irreducible domain $\mathscr{T}_{r}=\mathbf{I J K}$ where the vertices are obtained by joining the irreducible two phase saturations.

The effective saturation $\underline{S}_{i} \in[0,1]$ of fluid $i$ is equal to 1 at the vertex I and vanishes on the opposite side $\mathbf{J K}$ of the irreducible triangle $\mathscr{T}_{r}$ , Fig. 1. The constraint $\underline{S}_{\mathrm{w}}+\underline{S}_{\mathrm{o}}+\underline{S}_{\mathrm{g}}=1$ also holds for effective saturations, which are linear functions of the saturations, and can be simply expressed by means of vector products. For every current point $\mathbf{M}$ inside the triangle $\mathscr{T}_{r}$,

$\underline{S}_{\mathrm{w}}=\frac{|\mathbf{J K} \wedge \mathbf{J M}|}{|\mathbf{J K} \wedge \mathbf{J I}|}, \quad \underline{S}_{\mathrm{o}}=\frac{|\mathbf{I} \mathbf{M} \wedge \mathbf{I K}|}{|\mathbf{I J} \wedge \mathbf{I K}|}, \quad \underline{S}_{\mathrm{g}}=\frac{|\mathbf{I J} \wedge \mathbf{I M}|}{|\mathbf{I J} \wedge \mathbf{I K}|}$

The three denominators in equation (1) are equal to twice the area of the irreducible triangle $\mathscr{T}_{r}$.

The relative permeability of fluid $k$ can be obtained by weighting its contributions in two of the two phase systems by the partner effective

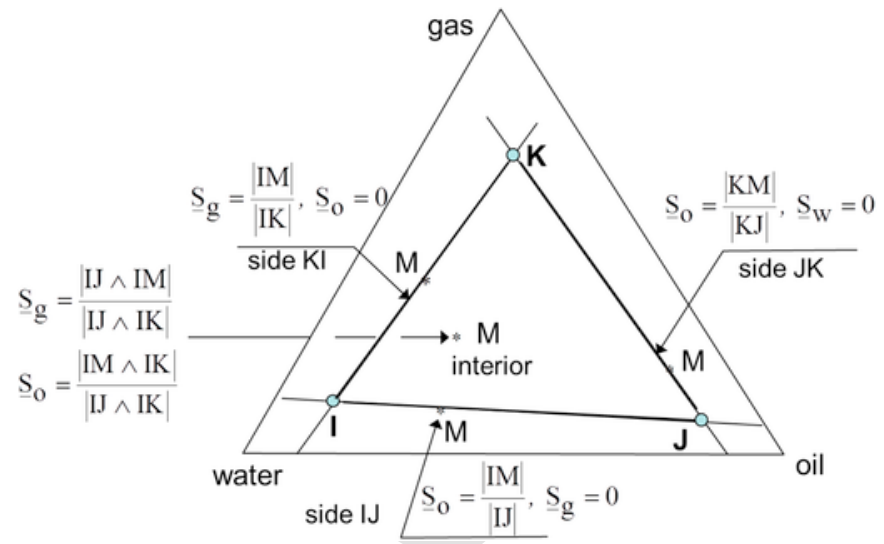

Fig. 1. Effective saturations in the interior and along the edges of the irreducible saturation triangle. The proposed expressions are continuous in the neighborhood of the edges and they retrieve the familiar two phase form along the edges. The third effective saturation is obtained by the condition that the three effective saturations sum to one.

saturations, namely along Hustad (2002),

$k_{r k}=\frac{k_{r k i} \underline{S}_{i}+k_{r k j} \underline{S}_{j}}{\underline{S}_{i}+\underline{S}_{j}}, \quad i \neq j \neq k$.

For each two phase system, the relative permeabilities are described along the lines of the Brooks-Corey model,

water/oil system, water wetting : $k_{r w o}=\underline{S}_{\mathrm{w}}^{2 / \lambda+3}, \quad k_{\text {row }}=$

oil/gas system, oil wetting : $\quad k_{r \mathrm{gg}}=\underline{S}_{\mathrm{o}}^{2 / \lambda+3}, k_{r \mathrm{go}}=$

water/gas system, water wetting : $k_{r \mathrm{wg}}=\underline{S}_{\mathrm{w}}^{2 / \lambda+3}, \quad k_{r \mathrm{gw}}=$

In the simulations, the pore size distribution index $\lambda$ is set to 0.8 which corresponds to a rather scattered pore size distribution. More details can be found in Appendix A, where the explicit forms (A.12) show that the contours of the relative permeabilities to water $k_{r \mathrm{w}}$ and oil $k_{r o}$ are parallel to the respective gas-oil and water-gas edges of the irreducible saturation triangle. The contours of the relative permeability to oil $k_{r o}$ are concave with respect to the oil vertex. A 3D representation of the relative permeabilities is shown on Fig. 2. The patterns are in overall agreement with the literature review over a number of sandstones and carbonates reported by (Egermann et al., 2013).

\subsection{Capillary pressures for a three-fluid phase porous medium}

The analysis is restricted to a water wet rock, where water is more wetting than oil which is more wetting than gas.

When considering the two phase system $i j$, where $j$ the wetting fluid, the capillary pressure $p_{c}=p_{c i j}=p_{i}-p_{j}$ is positive, it is infinite for $\underline{S}_{i}=1$, and vanishes for $\underline{S}_{i}=0$. The standard two phase relation uses the van Genuchten function,

$\frac{p_{c}}{p_{c 0}}=f\left(1-\underline{S}_{i}\right)=\left(\left(1-\underline{S}_{i}\right)^{\frac{-1}{m}}-1\right)^{\frac{1}{n}}$,

where $1>m=1-1 / n>0$. Now, for three-fluid phase systems, two alternative approaches are possible. A first approach involves postulating three capillary pressures. This approach seems attractive as specific information can be conveyed in each of the three capillary 

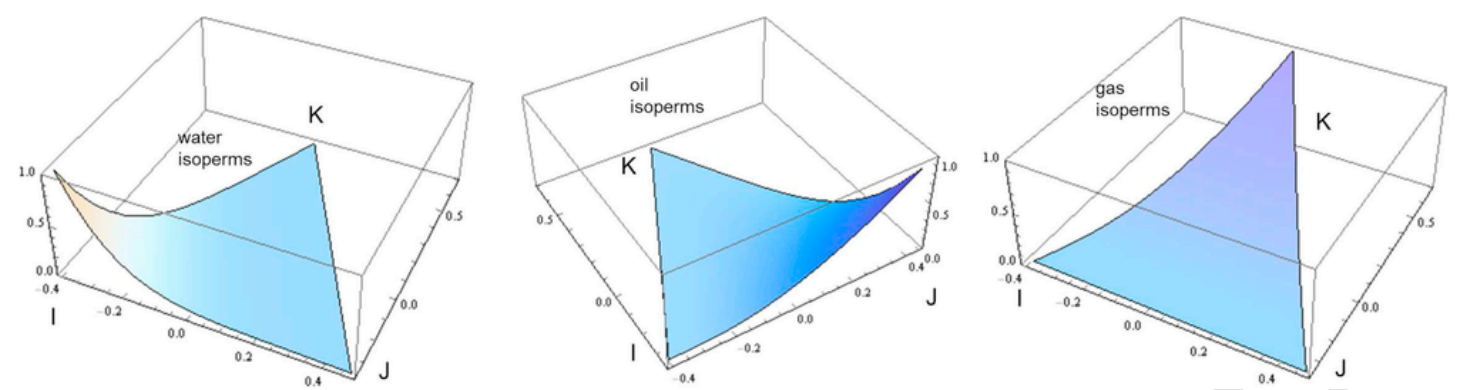

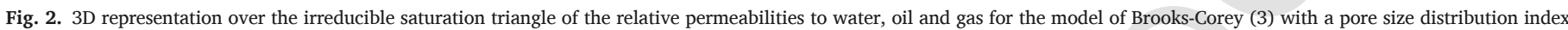
$\lambda=0.8$ based on the weighting (2).

pressures. Still, the latter need a priori to be corrected, as only two independent capillary pressures exist, since $p_{\mathrm{g}}-p_{\mathrm{w}}=\left(p_{\mathrm{g}}-p_{\mathrm{o}}\right)+\left(p_{\mathrm{o}}-p_{\mathrm{W}}\right)$. A method that considers this constraint has been proposed by Hustad (2002). Alternatively, one may postulate two capillary pressures, and deduce the third one by the above compatibility condition. The latter approach has been adopted here.

If used in a three phase context, expression (4) implies that capillary pressure contours are parallel to the $j k$ side of the irreducible triangle. Instead of expression (4), a set of more realistic, original relations have been adopted in this work to reproduce the overall literature trends, as follows

$$
\begin{array}{r}
p_{c o w}=p_{\mathrm{o}}-p_{\mathrm{w}}=A\left(\left(1-\underline{S}_{\mathrm{o}}\right)^{\frac{-1}{m}}-1\right)^{\frac{1}{n}}\left(\left(1-\underline{S}_{\mathrm{o}}\right) \underline{S}_{\mathrm{g}}\right. \\
p_{c g o}=p_{\mathrm{g}}-p_{\mathrm{o}}=C\left(\left(1-\underline{S}_{\mathrm{g}}\right)^{\frac{-1}{m}}-1\right)^{\frac{1}{n}}\left(1-\underline{S}_{\mathrm{o}}+0.25(1-\right. \\
p_{c g w}=p_{\mathrm{g}}-p_{\mathrm{w}}=p_{c \mathrm{ow}}
\end{array}
$$

The parameters featuring in the above expressions are chosen to mimic the known features of a capillary curve along the edges of the saturation triangle where only two fluids are present (see (Gajo et al., 2017)), namely, $m=1-1 / n$, exponent $n=4$, and coefficients $A=30 \mathrm{kPa}, B=100 \mathrm{kPa}$ and $C=40 \mathrm{kPa}$.

A 3D representation of these capillary pressures is displayed in Fig. 3. The direction of saturation change has not been accounted for here, that is, no distinction is made between drainage and imbibition curves.

\subsection{Remark: pressure driven scheme for the three phase model}

While the effective saturation can be obtained by analytically inverting expression (4) in a two phase context, inverting the system of equation (5) for the saturations in a three phase context appears more complex. In fact, only two effective saturations and two capillary pressures are independent out of three, thus it is sufficient to consider any two of the three equation (5) to obtain any two increments of effective saturations $d \underline{\mathbf{S}}=\left(d \underline{S}_{\mathrm{w}}, d \underline{S}_{\mathrm{o}}, d \underline{S}_{\mathrm{g}}\right)$ in terms of the three incremental pressures $d \mathbf{p}=\left(d p_{\mathrm{w}}, d p_{\mathrm{o}}, d p_{\mathrm{g}}\right)$.

The above procedure is continuously used in the mixed FE formulation described below, where the pressures are the primary variables. The smoothness of the capillary-saturation curves is therefore of considerable importance in the convergence rate of the iterative process that is required to ensure the conservation of the fluid masses. Appendix A provides details on the algebraic aspects of this issue.

\section{Mechanical, transport and field equations}

The constitutive and field equations are outlined in this section, bearing in mind that, in space dimension $n_{s d}$, the $n_{s d}+3$ primary variables in the FE formulation are the $n_{s d}$ components of the displacement vector and the three phase scalar representatives (namely, either the three pressures, or a pressure and two saturations).

In the following, subscript 0 denotes a reference state. Summation over repeated mute indices does not apply unless explicitly stated.

\subsection{Mass balance}

The balance of mass of species $k$ states that the change of mass is due to diffusion through the solid skeleton and to mass transfer to/from the other species. With $d / d t$ denoting the time derivative following the solid skeleton,

$\frac{1}{J} \frac{d m_{k}}{d t}=-\operatorname{div} \mathbf{M}_{k}+n_{k} \hat{\rho}_{k}$,

where $\mathbf{M}_{k}=n_{k} \rho_{k}\left(\mathbf{v}_{k}-\mathbf{v}_{\mathrm{s}}\right)$ is the mass flux per unit surface of porous medium relative to the solid skeleton, $\mathbf{v}_{k}$ being the velocity of species $k$, $n_{k} \hat{\rho}_{k}$ is the rate of mass supply to species $k$ by the rest of the mixture, and $m_{k}=M_{k} / V_{0}$ is the mass content, i.e. the mass of the species relative to the initial volume of the porous medium, which may be expressed as the product of four familiar quantities
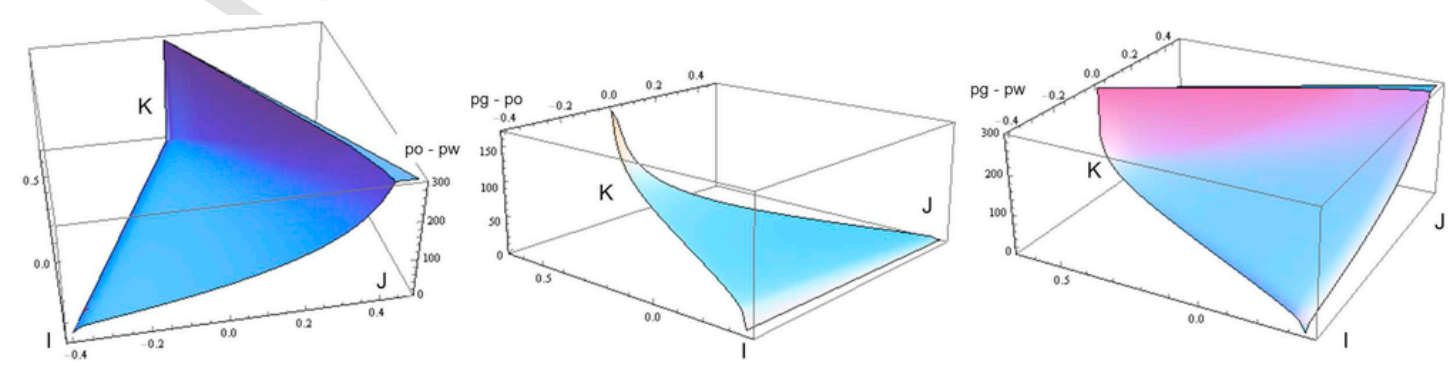

Fig. 3. 3D representation of the capillary pressures (5) over the irreducible saturation triangle for a water wet rock [ unit: kPa ] clipped at 300 kPa. Axes are not to scale. 
$m_{k}=J \rho_{k} n S_{k}$

with $J=V / V_{0}$ the Jacobian (i.e. the ratio of the current over initial volumes of the porous medium) and $\rho_{k}=M_{k} / V_{k}$ is the intrinsic mass density referred to the current volume $V_{k}$ of the species itself. The volume fraction $n_{k}=V_{k} / V$ refers to the current volume $V$ of the mixture, whereas the volume content $v_{k}=V_{k} / V_{0}$ refers to the initial volume (like the mass content). These quantities satisfy the relations $v_{k}=m_{k} / \rho_{k}=J n_{k}$. The porosity is the sum of volume fractions over fluid species, namely $n=\sum_{k \in \mathscr{K}_{f}} n_{k}$, and therefore $n_{\mathrm{s}}+n=1$.

\subsection{Constitutive equations of the solid grains and solid skeleton}

Let $K_{\mathrm{S}}$ be bulk modulus [ unit: Pa ] of the solid grains. Admitting that the solid is neither eroded nor it undergoes mass increase $\left(\hat{\rho}_{\mathrm{s}}=0\right)$, its change of relative mass density,

$\frac{1}{\rho_{\mathrm{s}}} \frac{d \rho_{\mathrm{s}}}{d t}=-\frac{1}{v_{\mathrm{s}}} \frac{d v_{\mathrm{s}}}{d t}=\frac{1}{K_{\mathrm{s}}} \frac{d p_{\mathrm{s}}}{d t}$,

is then linearly related to its intrinsic mean stress $p_{\mathrm{s}}=-\frac{1}{3} \operatorname{tr} \sigma_{\mathrm{s}}$.

At the mixture level, the linear isotropic elastic equation links the strain $\varepsilon$ of the solid skeleton to the effective stress relative to the reference state,

$\sigma^{\prime}-\sigma_{0}^{\prime}=\mathbb{E}: \varepsilon=K \operatorname{tr} \varepsilon \mathbf{I}+2 \mu \operatorname{dev} \varepsilon$,

where $\mathbf{I}$ is the second order identity tensor, and $K$ and $\mu$ are the isothermal drained bulk and shear moduli [ unit: Pa ] of the solid skeleton. The effective stress tensor accounts for the fluid pressures via volume weighting,

$\sigma^{\prime}=\sigma+\kappa \bar{p} \mathbf{I}, \quad \kappa=1-\frac{K}{K_{\mathrm{s}}}, \quad \bar{p}=\sum_{k \in \mathscr{K}_{f}} S_{k} p_{k}$.

At this stage it is useful to record the relations between the total stress $\sigma=n_{\mathrm{s}} \sigma_{\mathrm{s}}-n \bar{p} \mathbf{I}$, the effective stress $\boldsymbol{\sigma}^{\prime}$, the intrinsic solid stress $\boldsymbol{\sigma}_{\mathrm{s}}$ and the average fluid pressure $\bar{p}$ :

$\sigma_{\mathrm{s}}=\frac{1}{n_{\mathrm{s}}}(\sigma+\bar{p} \mathbf{I})=\frac{1}{n_{\mathrm{s}}}\left(\sigma^{\prime}+(n-\kappa) \bar{p} \mathbf{I}\right)$.

Substituting these relations into (8) yields the rates of change of the volume content $v_{\mathrm{S}}$ and of the volume fraction $n_{\mathrm{S}}$ of the solid, namely to first order,

$\frac{d v_{\mathrm{s}}}{d t}=\frac{d\left(n_{\mathrm{s}} J\right)}{d t}=\frac{K}{K_{\mathrm{s}}} \frac{d}{d t} \operatorname{tr} \varepsilon+\frac{n-\kappa}{K_{\mathrm{s}}} \frac{d \bar{p}}{d t}$,

$-\frac{d n}{d t}=\frac{d n_{\mathrm{s}}}{d t}=(n-\kappa) \frac{d}{d t} \operatorname{tr} \varepsilon+\frac{n-\kappa}{K_{\mathrm{s}}} \frac{d \bar{p}}{d t}$.

The balance of momentum of the mixture as a whole is expressed in terms of the total stress $\boldsymbol{\sigma}$ and of the total body force $\rho \mathbf{g}$, where $\rho$ is the total mass density of the porous medium, and $\mathbf{g}$ the gravity vector,

$\operatorname{div} \sigma+\rho \mathbf{g}=0$.

\subsection{Equations of fluid transport}

The flow of fluid $k$ through the porous skeleton is described by Darcy's law, providing the mass flux $\mathbf{M}_{k}$ in terms of the pressure gradient,

$$
\begin{aligned}
\mathbf{M}_{k} & \equiv n_{k} \rho_{k}\left(\mathbf{v}_{k}-\mathbf{v}_{\mathrm{s}}\right) \\
& =-D_{k}\left(\nabla p_{k}-\rho_{k} \mathbf{g}\right), \quad D_{k} \\
& \equiv \rho_{k} k_{r k} \frac{k_{\text {in }}}{\eta_{k}} .
\end{aligned}
$$

In the above, the intrinsic hydraulic permeability $k_{\text {in }}$ [unit: $\mathrm{m}^{2}$ ] is a property of the porous skeleton while the dynamic viscosity $\eta_{k}$ [ unit: $\mathrm{Pa}$ $\times \mathrm{s}=\mathrm{kg} / \mathrm{m} / \mathrm{s}=10^{3}$ centipoise $\mathrm{cP}$ ] is a property (strongly temperature dependent for oil) of the permeant fluid $k$. If the whole pore space is filled by the sole fluid $k$, the relative permeability $k_{r k}$ [unit: 1 ] is equal to 1 ; otherwise, it is smaller than 1 and depends on the saturations of the fluids (and flow history) in the pore space, as detailed in Sect. 2.1.

The fluid flow is effectively described by the above equation provided that no dissolution of one fluid into the other (or mutual dissolution) takes place, i.e., the three fluids are assumed immiscible. While this assumption is suitable for (e.g.) the flow of water, oil and a hydrocarbon gas, it may be inadequate when considering the interaction of certain fluids such as water and air, oil and air or water and carbon dioxide. In such cases a rate equation, or other tools (like adsorption isotherms) for the dissolved/precipitated component should be adopted, and its transport addressed, e.g. via Fick's law.

\section{Finite element formulation}

The primary variables in the generic element $e$, namely the displacement of the solid skeleton and the three fluid pressures,

$\mathbb{X}^{e}=\left[\mathbf{U}^{e} \mathbf{P}_{\mathrm{w}}^{e} \mathbf{P}_{\mathrm{o}}^{e} \mathbf{P}_{\mathrm{g}}^{e}\right]^{\mathrm{T}}$

are interpolated through the respective shape functions,

$\mathbf{u}=\mathbf{N}_{u}^{e} \mathbf{U}^{e}, \quad p_{k}=\mathbf{N}_{p}^{e} \mathbf{P}_{k}^{e}, \quad k \in \mathscr{K}_{f}$.

The variations associated with the primary variables are interpolated with the same functions along a Galerkin scheme. Four node bilinear elements are used for the pressures while a Q8 quadratic interpolation is adopted for the displacement.

\subsection{Discretized mass balance equations of the pore fluids}

The balance of mass (6) of the fluid $k$ expressed in terms of the mass content $m_{k}$ is first discretized in time with a generalized trapezoidal scheme defined by parameter $\alpha \in[0,1]$. At step $n+1$, iteration $i$, the balance of mass is enforced at time $t_{n+\alpha}=t_{n}+\alpha \Delta t$, where $\Delta t=t_{n+1}-t_{n}$, namely

$\frac{1}{J_{n+\alpha}} \frac{m_{k, n+1}^{i}-m_{k, n}}{\Delta t}+\operatorname{div} \mathbf{M}_{k}+\widehat{f}_{k}=0$,

where $\hat{f}_{k}=-n_{k} \hat{\rho}_{k}$, the mass flux $\mathbf{M}_{k}$ is given by Darcy's law (15), and $m_{k, n+1}^{i}$ can be deduced from the primary variables through eqn. (7) (using eqns. (5) and (13)). All terms, except the finite difference of mass content, are estimated at $(n+\alpha, i)$. 
It is worth emphasizing that in eqn. (18) attention is laid in avoiding any linearization ${ }^{1}$ of the mass balance (and correspondingly in manipulating the residual), thus obtaining a formulation that is equivalent to the so-called mixed form of Richards' equation (for the balance equation of a single fluid in a two-fluids saturated porous medium (Bear and Verruijt, 1987),).

The element residual $\mathbf{R}_{k}^{e}$ at $(n+\alpha, i)$ contributed by the element $e$ is obtained by spatial integration of the weak form (no sum over repeated index $k$ ),

$\mathbf{R}_{k}^{e}=\mathbf{D}_{k}^{e} \mathbf{X}_{k}^{e}+\mathbf{K}_{k k}^{e} \mathbf{P}_{k}^{e}+\mathbf{E}_{k}^{e}+\mathbf{L}_{k}^{e}$,

with

$$
\begin{aligned}
& \mathbf{D}_{k}^{e}=\overbrace{\int_{V_{e}}\left(\mathbf{N}_{p}^{e}\right)^{\mathrm{T}} \mathbf{N}_{p}^{e} d V_{e}}^{\text {lumped }} \\
& \mathbf{K}_{k k}^{e}=\quad \int_{V_{e}}\left(\mathbf{B}_{p}^{e}\right)^{\mathrm{T}} D_{k} \mathbf{B}_{p}^{e} d V_{e}, \\
& \mathbf{E}_{k}^{e}=\int_{V_{e}}-\left(\mathbf{B}_{p}^{e}\right)^{\mathrm{T}} D_{k} \rho_{k} \mathbf{g}+\left(\mathbf{N}_{p}^{e}\right)^{\mathrm{T}} \hat{f}_{k} d V_{e} \\
& \mathbf{L}_{k}^{e}=\int_{\partial V_{e}}\left(\mathbf{N}_{p}^{e}\right)^{\mathrm{T}} j_{k} d S_{e},
\end{aligned}
$$

with $\stackrel{2 \times 4}{\mathbf{B}_{p}^{e}}=\nabla \mathbf{N}_{p}^{e}$ and with $\stackrel{4 \times 1}{\mathbf{X}_{k}^{e}}$ denoting the vector containing the variation of mass content of $k$ - th fluid, computed at each element node, namely each nodal component of $\mathbf{X}_{k}^{e}$ is equal to

$X_{k}=\frac{1}{J_{n+\alpha}} \frac{m_{k, n+1}-m_{k, n}}{\Delta t}$.

The notation $j_{k}=\mathbf{M}_{k} \cdot \mathbf{n}$ refers to the normal mass flux on the boundary of outward unit normal $\mathbf{n}^{2}$. Storage terms in eqn. (21) are lumped in order to obtain a regularized solution (without spurious oscillation), as proposed by Celia et al. (1990). The evaluation and the lumping of the matrix $\mathbf{D}_{k}^{e}$ has been performed with ordinary numerical Gaussian quadrature, followed by the row sum technique.

As is discussed in Sect. 7.1, when dealing with co-current flow simulations, the particular boundary conditions arising from the co-existence of multiple, non-miscible fluids, can be conveniently treated with an ad-hoc, convective-like, boundary condition, namely $j_{k}=k_{k, c}\left(p_{k}-p_{k, e x t}\right)$. In this case, the convective term contributes to the stabilization of the diffusion matrix as it has exactly the same for-

1 The relations.

$$
(a b)^{1}-(a b)^{0}=\left(a^{1}-a^{0}\right) b^{0}
$$

$(a b c)^{1}-(a b c)^{0}=\left(a^{1}-a^{0}\right) b^{1} c^{1}+a^{0}(t$ $(a b c d)^{1}-(a b c d)^{0}=\left(a^{1}-a^{0}\right) b^{1} c^{1} d^{1}+a^{0}\left(b^{1}-b^{0}\right) c^{1}$

applied to eqn. (18), with the mass content defined in eqn. (7), show that the expansion (28) introduces errors unless the coefficients are estimated exactly as indicated in eqn. (19). The above way of estimating residuals is consistent with eqn. (7), and may be referred to as 'expanded form' or 'non-linearized form' of the residuals.

2 Alternatively, if the body term is not integrated by parts, it disappears from the flux $j_{k}$ and the integrand in $\mathbf{E}_{k}^{e}$ turns to $\left(\mathbf{N}_{p}^{e}\right)^{\top} \operatorname{div}\left(D_{k} \rho_{k} \mathbf{g}\right)$. mat and proper sign,

$$
\begin{aligned}
& \mathbf{K}_{k k}^{e} \rightarrow \mathbf{K}_{k k}^{e}+\int_{\partial V_{e}}\left(\mathbf{N}_{p}^{e}\right)^{\mathrm{T}} k_{k, c}\left(\mathbf{N}_{p}^{e}\right) d S_{e} \\
& \mathbf{L}_{k}^{e} \rightarrow \mathbf{L}_{k}^{e}+\int_{\partial V_{e}}\left(\mathbf{N}_{p}^{e}\right)^{\mathrm{T}} k_{k, c}\left(-p_{k, \text { ext }}\right) d S_{e}
\end{aligned}
$$

\subsection{Discretized balance of momentum}

The momentum balance is discretized in the standard predictor corrector format yielding the element residual at step $n+\alpha$, iteration $i$,

$$
\left(\mathbf{R}_{u}^{e}\right)_{n+\alpha}^{i}=\int_{V_{e}}\left(\mathbf{B}_{u}^{e}\right)^{\mathrm{T}} \sigma_{n+\alpha}^{i} d V_{e}+\left(\mathbf{E}_{u}^{e}\right)_{n+\alpha}^{i}+\left(\mathbf{L}_{u}^{e}\right)_{n+\alpha}^{i}
$$

with

$$
\begin{aligned}
\underset{\widetilde{\mathbf{E}}_{u}^{e}}{16 \times 1} & =\int_{V_{e}}\left(\mathbf{N}_{u}^{e}\right)^{\mathrm{T}}(-\rho \mathbf{g}) d V_{e}, \\
\underset{16 \times 1}{\widetilde{\mathbf{L}}_{u}^{e}} & =\int_{\partial V_{e}}\left(\mathbf{N}_{u}^{e}\right)^{\mathrm{T}}(-\mathbf{t}) d S_{e} .
\end{aligned}
$$

Here $\mathbf{t}=\boldsymbol{\sigma} \cdot \mathbf{n}$ is the total traction. The residuals are estimated at the predictor value of the time marching.

\subsection{Consistent linearization for the algorithmic tangent operator}

A first order expansion of the diffusion and convection terms in the residual (20) results in the diffusion tangent matrices estimated at $(n+\alpha, i)$,

${ }_{t} \mathbf{K}_{k j}^{e}={ }_{t 1} \mathbf{K}_{k j}^{e}+{ }_{t 2} \mathbf{K}_{k j}^{e}+{ }_{t 3} \mathbf{K}_{k j}^{e}+{ }_{t 4} \mathbf{K}_{k j}^{e}$,

where

$$
\begin{gathered}
{ }_{t 1} \mathbf{K}_{k j}^{e}=\int_{V_{e}}\left(\mathbf{B}_{p}^{e}\right)^{\mathrm{T}}\left(D_{k} I_{k j} \mathbf{B}_{p}^{e}+\frac{\partial D_{k}}{\partial p_{j}}\left(\mathbf{B}_{p}^{e} \mathbf{P}_{k}^{e}\right) \mathbf{N}_{p}^{e}\right) d V_{e} ; \\
{ }_{t 2} \mathbf{K}_{k j}^{e}=\int_{V_{e}}\left(-\left(\mathbf{B}_{p}^{e}\right)^{\mathrm{T}}\left(D_{k} \frac{I_{k j}}{K_{k}}+\frac{\partial D_{k}}{\partial p_{j}}\right) \rho_{k} \mathbf{g}+\left(\mathbf{N}_{p}^{e}\right)^{\mathrm{T}} \frac{\partial \hat{f}_{k}}{\partial p_{j}}\right) \mathbf{N}_{1} \\
{ }_{t 3} \mathbf{K}_{k j}^{e}=\int_{\partial V_{e}}\left(\mathbf{N}_{p}^{e}\right)^{\mathrm{T}}\left(k_{k, c} \mathbf{N}_{p}^{e} I_{k j}+\frac{\partial k_{k, c}}{\partial p_{j}}\left(\mathbf{N}_{p}^{e} \mathbf{P}_{k}^{e}\right) \mathbf{N}_{p}^{e}\right) d S_{e} \\
{ }_{t 4} \mathbf{K}_{k j}^{e}=\int_{\partial V_{e}}\left(\mathbf{N}_{p}^{e}\right)^{\mathrm{T}} \frac{\partial k_{k, c}}{\partial p_{j}}\left(-p_{k, e x t}\right) \mathbf{N}_{p}^{e} d S_{e}
\end{gathered}
$$

and $I_{k j}=1$ for $j=k, I_{k j}=0$ for $j \neq k$.

While attention is laid in avoiding any linearization in manipulating the residual as indicated in eqn (20), the algorithmic (tangent) matrix used to drive the iterative process is obtained by linearization of this residual, while strictly keeping the lumped structure (see Sects 3 and 4.1). A consistent tangent has been implemented; however, when the saturation of one phase is close to the irreversible values, some terms of the tangent matrix that could assume extreme values have been adjusted, as described in Section 6.4.

Expansion to first order of the storage term in the balance of mass for the fluid $k$, eqn (18) introduces the terms,

$d_{k u} \delta \operatorname{tr} \varepsilon+\sum_{j \in \mathscr{K}_{f}} d_{k j} \delta p_{j}$, 
where again $\delta$ denotes the difference between two iterations and

$$
\begin{aligned}
d_{k u} & =\rho_{k} S_{k} \kappa, \quad d_{k j} \\
& =n \rho_{k}\left(\frac{S_{k}}{K_{k}} I_{k j}-\frac{S_{k}}{n} \frac{n-\kappa}{K_{\mathrm{s}}} \frac{\partial \bar{p}}{\partial p_{j}}+\frac{\partial S_{k}}{\partial p_{j}}\right), \quad k, j \\
& \in \mathscr{K}_{f} .
\end{aligned}
$$

The isothermal bulk moduli $K_{k}=d p_{k} / \operatorname{Ln} \rho_{k}$ of the liquids $(k=\mathrm{w}, \mathrm{o})$ are assumed to remain constant over the time step, while the isothermal bulk modulus of the gas is equal to the absolute gas pressure. The derivatives required in (27) and (29) are detailed in Appendix A.

The capacity matrices $\mathbf{D}_{k u}^{e}$ and $\mathbf{D}_{k j}^{e}$ are defined as follows

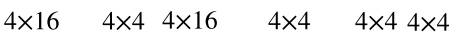

$\mathbf{D}_{k u}^{e}=\mathbf{D}_{k}^{e} \quad \mathbf{B}_{u}^{*}, \quad \mathbf{D}_{k j}^{e}=\mathbf{D}_{k}^{e} \boldsymbol{\Delta}_{k j}, \quad k, j \in \mathscr{K}_{f}$

where $\mathbf{D}_{k}^{e}$ is the lumped matrix defined in eqn. (21a), $\mathbf{B}_{u}^{*}$ is the matrix relating the product of $d_{k u}$ times the volumetric strain evaluated at each corner node to the nodal displacements and $\Delta_{k j}$ is a diagonal, $4 \times 4$ matrix having at each diagonal position, the value of $d_{k j}$ evaluated at the corresponding corner node.

The contribution of the balance of momentum to the algorithmic tangent matrix is obtained by consistent linearization,

$$
\left(\mathbf{R}_{u}^{e}\right)_{n+\alpha}^{i}=\left[\alpha \Delta t_{t} \tilde{\mathbf{K}}_{u u}^{e} \quad \alpha \Delta t_{t} \tilde{\mathbf{K}}_{u w}^{e} \quad \alpha\right.
$$

with $\mathbb{V}^{e}$ the time derivative of the primary unknown vector $\mathbb{X}^{e}$ defined by (16), and

$$
\begin{aligned}
& \widetilde{\mathbf{K}}_{u u}^{16 \times 16}=\quad \int_{V_{e}}\left(\mathbf{B}_{u}^{e}\right)^{\mathrm{T}} \mathbf{D}^{\mathrm{el}} \mathbf{B}_{u}^{e} d V_{e}, \\
& { }_{t}^{16 \times 4} \widetilde{\mathbf{K}}_{u k}^{e}=\int_{V_{e}}\left(\operatorname{tr} \mathbf{B}_{u}^{e}\right)^{\mathrm{T}}\left(-\kappa \frac{\partial \bar{p}}{\partial p_{k}}\right) \mathbf{N}_{p}^{e} d V_{e}, \quad k \in \mathscr{K}_{f},
\end{aligned}
$$

Here $\mathbf{D}^{\mathrm{el}}$ is the elastic stiffness matrix. The matrices are estimated at the predictor value of the time marching scheme denoted by the superimposed symbol.

Gathering the residuals of the balance of momentum and balances of mass for the whole set of elements provides the system,

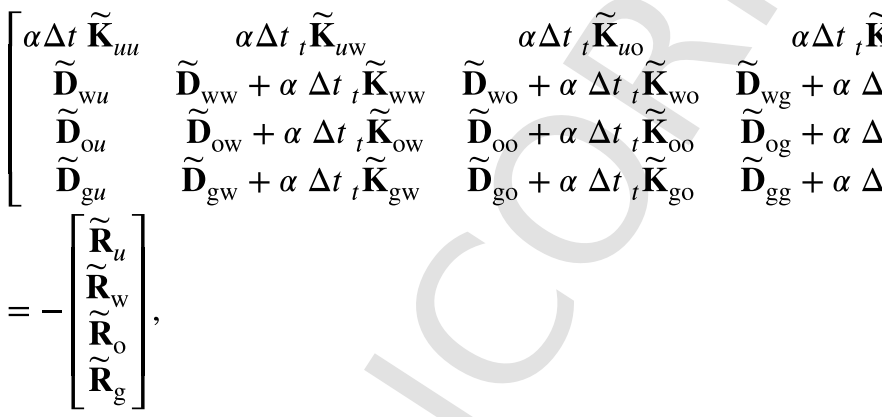

to be solved at each iteration $i$ for increment $\Delta \mathbb{V}=\mathbb{V}_{n+1}^{i}-\mathbb{V}_{n+1}^{i-1}$.

The constitutive equations for the capillary pressures and relative permeabilities and the time marching scheme were introduced in a Fortran domestic finite element code, and numerical integration was performed using a backward Euler scheme (with $\alpha=1$ ). Moreover, the applications did not involve mass exchanges, thus $\hat{\rho}_{k}=0$.
It is worth remarking that although no ad-hoc stabilization technique is adopted below (except for the lumping of storage terms, proposed by Celia et al. (1990)), the computed saturation plots do not show wiggles and remain perfectly smooth.

In order to illustrate the physical aspects of the problem first, computational aspects are addressed in Sect. 6, after the description of gas injection simulations (Sect. 5). The analysis of computational aspects will be limited to the problem of gas injection, for space reasons.

\section{Gas injection simulations}

The above outlined computational framework is used in this section to describe a drainage process in a water-wet deformable rock core, in which the least wetting phase (gas) displaces the intermediate and most wetting phases, namely oil and water respectively.

Although the core flooding simulations shown hereafter are essentially one dimensional processes, they were run with 2D (plane strain) elements. It should be remarked that no aspect of the constitutive equations, nor of the computational time marching scheme, is restricted to 1D. In fact some 2D simulations were performed with skewed elements to analyse the effects of element distortion (see Sect. 6.7). Besides, all aspects of the constitutive equations described above (mechanics, transport, capillary pressures and relative permeabilities) apply to a 3D context.

Core flooding tests are simulated, since they are routinely performed by petroleum engineers, and there is a relative abundance in the literature of laboratory experimental data. However, since typically the outputs of published tests lack generality, and the test details are often missing, no attempt was made to reproduce a particular published experiment. Instead, the qualitative predictions of the constitutive model and the capabilities and robustness of the computational scheme were explored in a more systematic way by referring to more general, albeit typical, testing conditions.

\subsection{Initial and boundary conditions}

Gas flooding of a deformable core of length $L=0.3 \mathrm{~m}$ is considered. The initial saturation with water and oil is uniform in space as indicated by Fig. 4. The mass densities and volume fractions of the involved fluids are shown in Table 1.

Gas injection at the inlet $(x=L)$ starting at time $t=0^{+} \mathrm{s}$ is simulated adopting the hydraulic boundary conditions shown in Fig. 5. At the outlet $(x=0)$, the core is brought in contact with a water reser-

$$
\begin{aligned}
& S w=0.10 ; p w 0=100 \mathrm{kPa} \\
& \text { So }=0.90 ; \quad p o=p w 0+p \operatorname{cow}\left(S_{w}, S_{o}, S g\right)=355 \mathrm{kPa} \\
& \mathrm{Sg}=0 ; \quad \mathrm{pg}=\mathrm{pw0}+\mathrm{pcgw}(\mathrm{Sw}, \mathrm{So}, \mathrm{Sg})=355 \mathrm{kPa} \\
& \text { core } \\
& \mathrm{x}=\mathrm{L}=0.3 \mathrm{~m}
\end{aligned}
$$$$
\mathrm{x}=0
$$

Fig. 4. Initial conditions for a 1D gas injection test. The saturations are prescribed uniform over the core. The pressures and capillary pressures are deduced from the saturations based on the constitutive relations.

Table 1

Initial mass densities and volume fractions of fluids. The initial porosity (total volume fraction of the three fluids) is equal to 0.4 .

\begin{tabular}{lllc}
\hline & water & oil & gas \\
\hline mass densities $\left(\mathrm{kg} / \mathrm{m}^{3}\right)$ & $\rho_{\mathrm{W}}=1000$ & $\rho_{\mathrm{o}}=1010$ & $\rho_{\mathrm{g}}=1$ \\
volume fractions & $n_{\mathrm{W}}=0.24$ & $n_{\mathrm{o}}=0.16$ & $n_{\mathrm{g}}=0.0$ \\
\hline
\end{tabular}




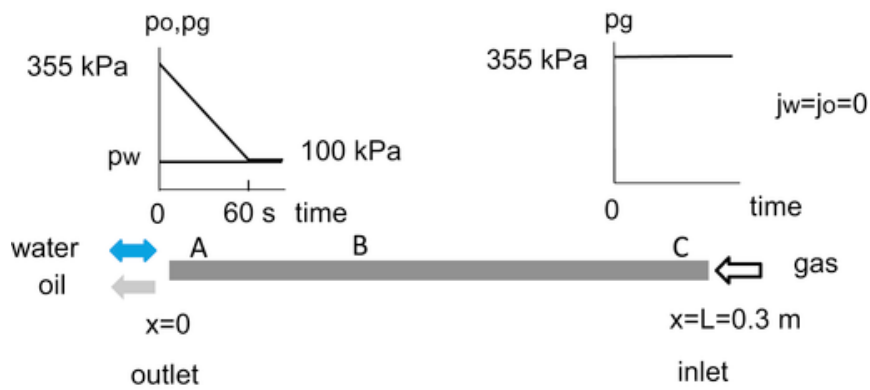

Fig. 5. Boundary conditions for a 1D core gas injection test. At the outlet, (a) initially the pressure of the wetting fluid (water) is assumed to be equal to the pressure of the outlet water reservoir; (b) the oil and gas pressures are reduced to the water pressure in a short time interval at the end of which the specimen at $x=0$ is water saturated. At the inlet, gas injection is pressure controlled and the water and oil fluxes, $j_{\mathrm{W}}$ and $j_{\mathrm{o}}$ respectively, are null.

voir: this process is simulated by decreasing, in a short time interval, the pressures of both oil and gas to reach the same pressure as water (Fig. 5 ), leading the outlet water saturation to $S_{\mathrm{W}}=1.0$. At the same time, at the inlet $(x=L)$, the gas pressure is kept constant and the water and oil fluxes are set to zero.

The mechanical boundary conditions are such that the lateral displacement is set to vanish all along the core, while the axial displacement at the inlet $(x=L)$ is set to zero. At the outlet $(x=0)$, the total traction $\sigma_{x x}(x=0, t)$ is maintained fixed to the initial average fluid pressure $-\kappa \bar{p}(x=0, t=0)$. As far as sign conventions are concerned, compressive stress and contractive strain are taken negative.

The transport and mechanical constitutive parameters adopted are listed in Tables 2 and 3 respectively.

The mesh is refined in the neighborhood of the outlet where the axial element size is progressively reduced to $1 / 8$ of its value at core center. The storage terms of the residuals and tangent matrix are estimated at $n_{\mathrm{G}} \times n_{\mathrm{G}}$ Gauss points. As a standard value $n_{\mathrm{G}}=2$ is used, but also the effect of a larger number (within the range $n_{\mathrm{G}}=2-4$ ) has been tested.

The simulations require to set a minimum value $k_{r}^{\mathrm{min}}$ for the relative permeabilities. In the simulations shown below, the following reference parameters are used: fine mesh counting 63 elements (see Sect. 6.1), minimum relative permeability $k_{r}^{\mathrm{min}}=10^{-4}$, intrinsic permeability $k_{\text {in }}=1$ darcy. The injection rate and some material parameters will be altered to test the sensitivity of the three-fluid phase system to their change. The expressions of the capillary pressures presented in Sect. 2.2 are adopted throughout. The irreducible saturations are all set to zero in this section. Also the mass supplies are set to zero, assuming for

Table 2

Intrinsic permeability of a reservoir rock and viscosities at ambient temperature.

\begin{tabular}{llll}
\hline intrinsic & viscosity & & \\
\hline permeability & water & oil & gas \\
$10^{-12} \mathrm{~m}^{2}$ & $1 \mathrm{cP}$ & $2 \mathrm{cP}$ & $0.01 \mathrm{cP}$ \\
\hline
\end{tabular}

Table 3

Bulk moduli of the solid skeleton and constituents, except gas which is considered perfect.

\begin{tabular}{llllc} 
& $\begin{array}{l}\text { solid } \\
\text { skeleton }\end{array}$ & $\begin{array}{l}\text { solid } \\
\text { grains }\end{array}$ & water & oil \\
\hline $\begin{array}{l}\text { bulk modulus } \\
(\mathrm{GPa})\end{array}$ & 0.555 & 36 & 2.2 & 1 \\
\hline
\end{tabular}

simplicity that the solid and the three fluids do not undergo mass loss/ gain by exchange with other constituents and/or with the neighborhood.

\subsection{Three phase numerical results}

In Fig. 6 spatial profiles of water, oil and gas pressure and saturation are shown for gas injection simulations. It should be noted that the dotted curves in Fig. 6-(a) correspond to a vanishing gas saturation, so that the gas pressure and the oil-gas capillary pressure turn elusive. In other words, the dotted lines are outputs stemming from formally solving the independent gas equation. Gas injection is controlled by decreasing the outlet gas pressure in a short period of time while keeping the inlet gas pressure at its initial value. This boundary signal propagates into the rock through a saturation front, that becomes steeper as it reaches the outlet at gas breakthrough, Fig. 6. After breakthrough, the saturation profiles are smooth along the core. In fact, the saturation front is relatively modest and most of the changes of saturation take place progressively along smooth profiles.

The pressure profiles of oil and water establish progressively and monotonously. Since the saturations are initially uniform, so are the relative permeabilities. Moreover, since the pressures at the boundaries reach their final values quickly, the pressure profiles of oil and gas remain linear on the outlet side until they are perturbed by the permeability changes originated at the inlet. As the core is put in contact with the reservoir, the water saturation close to the outlet increases sharply. At some distance, however, the water saturation remains closer to its initial low value: the water pressure decreases accordingly, to temporarily maintain large consistent capillary pressures. Retrieving a smooth monotonous profile takes as long for the water pressure as for the oil and gas pressures.

The water saturation is virtually unaffected by the gas injection over most of the core length, except for the vicinity of the outlet, Figs. 6 and 7-(b).

The total traction applied at the outlet is maintained to its initial value. As a consequence, the effective stress $\sigma_{x x}^{\prime}(x=0, t)=\kappa(\bar{p}(x=0, t)-\bar{p}(x=0, t=0))$, which is initially zero, becomes compressive as the specimen is put in contact with water and the oil and gas pressures are reduced from $355 \mathrm{kPa}$ to $100 \mathrm{kPa}$. The pressures are also reduced along the core so that the strain is expected to be contractive at any position, Fig. 7-(a).

The saturation path during injection highlights the two steps of pressure change at the boundaries and subsequent flow, Fig. 7-(b). The contact of the core with the reservoir at early times is apparent at point A $(x=0.01 \mathrm{~m})$ where water saturation increases until gas breakthrough: from that moment onward, gas injection results mostly in oil production but also in some water production. Along most part of the core, points $\mathrm{B}(x=0.1 \mathrm{~m})$ and $\mathrm{C}(x=0.29 \mathrm{~m})$, gas injection does not change water saturation, Figs. 6 and 7-(b). The associated changes of relative permeabilities and capillary pressures along the paths can be appreciated from Figs. 2 and 3.

Sensitivity of the water pressure profiles to initial saturations was explored, but it is not shown here for brevity. Although the gradient of water pressure at outlet is smooth, the profile of the water pressure exhibits a front which becomes very steep as the initial water saturation decreases, or equivalently as the initial oil saturation increases. Correspondingly, the propagation speed of the front increases and the water flux at outlet exhibits a spike with an increasingly large amplitude.

Further, changing the intrinsic permeability results in expected effects on gas saturation profiles and on oil mass outflow, i.e. the larger the intrinsic permeability, the faster the propagation of the gas front. Similarly, increasing the gas pressure results in a faster gas front. 

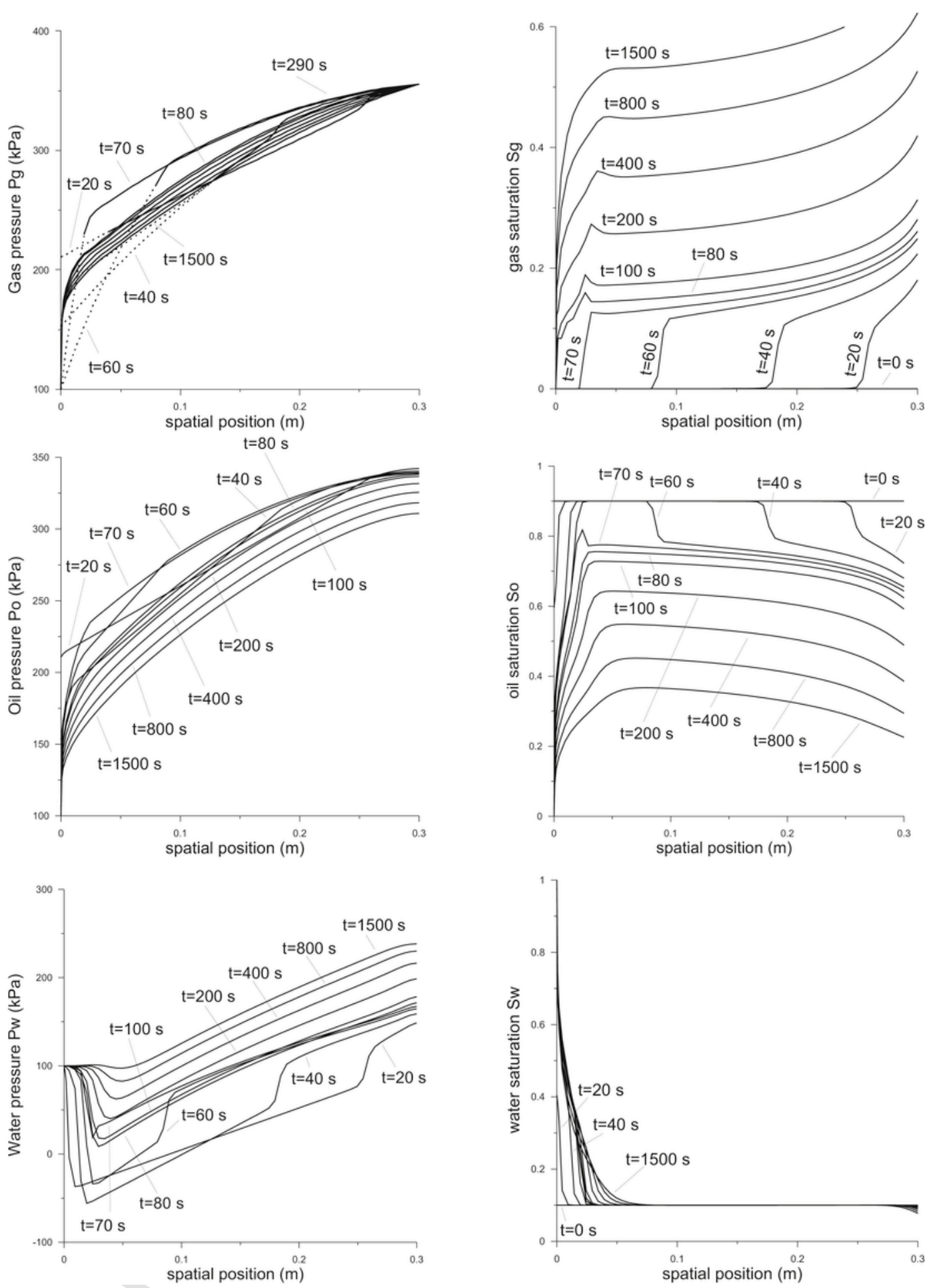

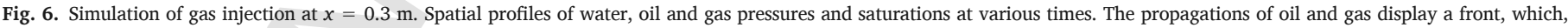
although smooth, tend to steepen progressively over time until gas breakthrough. The dotted curves correspond to a vanishing gas saturation.

\section{Computational details of gas injection simulation}

While the simulations shown in Sect. 5.2 have been obtained with reference parameters, in this section the sensitivity of the simulations to computational parameters is explored.

\subsection{Influence of mesh refinement}

Two main mesh types are tested: at the core center, the element lengths are equal to $2 \mathrm{~cm}$ for the coarse mesh counting 18 elements and $0.5 \mathrm{~cm}$ for the fine (reference) mesh counting 63 elements (with an aspect ratio of 4). In addition, a third mesh type including skewed elements, based on the reference mesh, was used to test the influence of a 


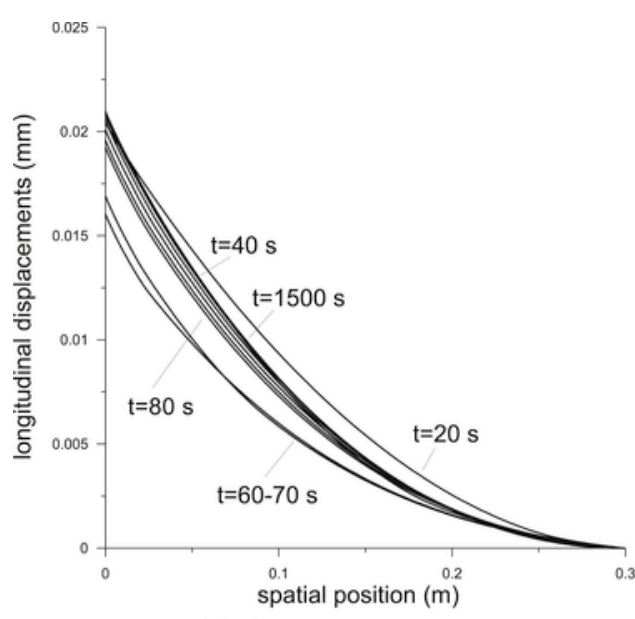

(a) Core contraction

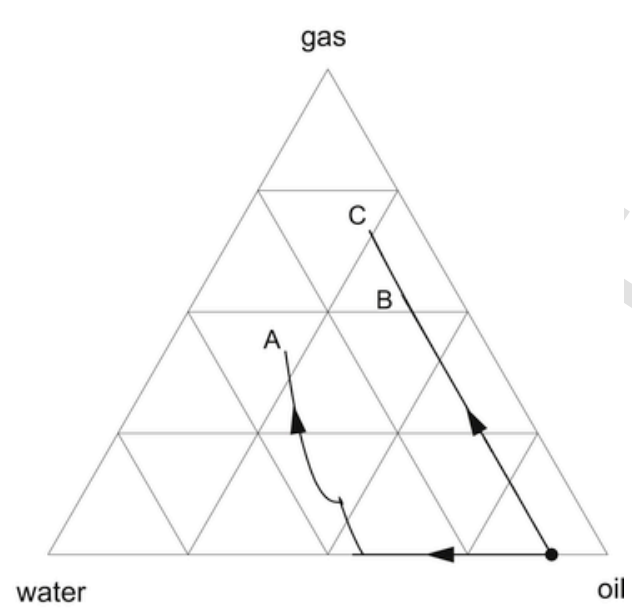

(b) Saturation path

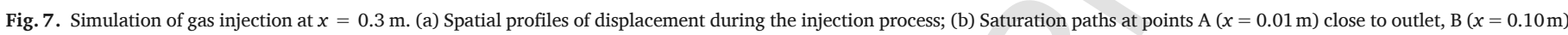

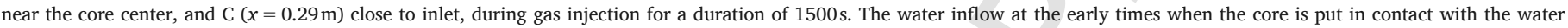
reservoir at outlet is clearly apparent at point A.

distorted mesh, as further discussed in Sect. 6.7. The different mesh types are shown in Fig. 9. The meshes are refined at the outlet, with a maximum element length enhancement of $1 / 8$. The coarse mesh was found to induce some oscillations of the propagation front close to the outlet, as can be seen in Fig. 8. However, these oscillations do not modify the rate of advancement of the front. Moreover, they fade out with time in such a way that, after 400 s of gas injection, the gas saturation profiles for the two meshes overlap.

The time evolution of the oil flux at outlet was also computed (not shown here for the sake of brevity), showing two maxima, the first of which is due to the sample being suddenly put in contact with the water reservoir, the second peak corresponding to the breakthrough. At early times $(<50 \mathrm{~s})$, the space profiles of the pressures are not far from their initial linear pattern, associated with uniform saturation profiles, Fig. 6. Therefore at early times, the fluxes are almost mesh independent. At later times, the gas saturation and water pressure pro-

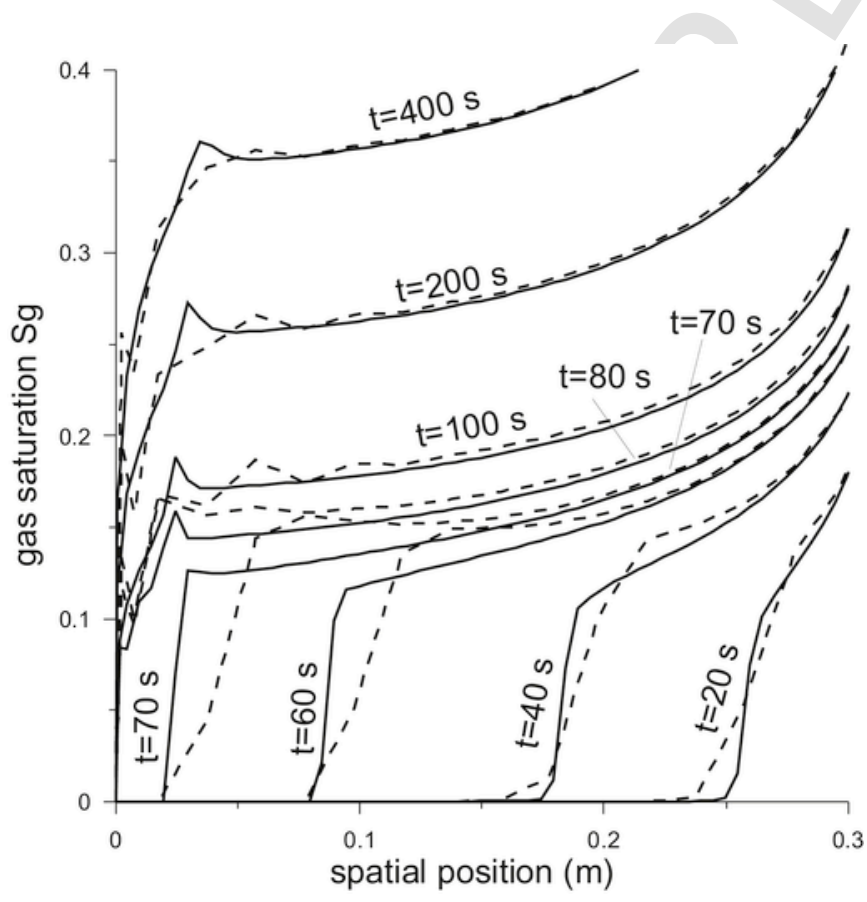

Fig. 8. Simulation of gas injection at $x=0.3 \mathrm{~m}$. Influence of mesh size: fine mesh (solid line) and coarse mesh (dashed line).

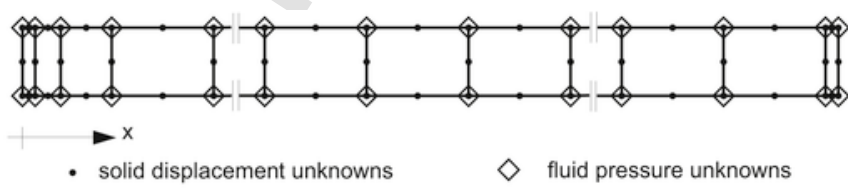

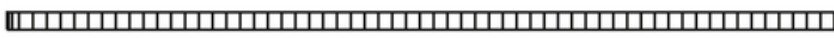

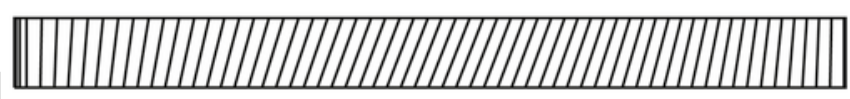

Fig. 9. Mesh types used in the simulations. The top figure shows an enlargement of the coarse mesh, in which the different symbols denote nodal points where solid displacement and/or fluid pressure unknowns are evaluated. The middle figure shows the fine mesh, used as a reference in this work, and the bottom figure shows the distorted mesh, based on the fine mesh, used to test the influence of element distortion on computed results as discussed in Sect. 6.7.

files remain smooth as long as the gas front has not reached the left end (where water pressure is controlled). In contrast, the profiles of both oil saturation and oil pressure become sharp. Consequently, the oil outflow is sensitive to the mesh size due to the fact that the fluxes are estimated at the centroid of the first/last element while all other integrals are estimated through $n_{\mathrm{G}} \times n_{\mathrm{G}}$ Gauss points.

\subsection{Number of Gauss points used to sample the diffusion contributions}

The number of Gauss points used to sample the diffusion contributions does not affect significantly the results, as shown in Fig. 10-(a). On the other hand, this number has a significant impact on the iterative process. In some cases, convergence was not achieved for a too low sampling number, and, in any case, increasing the number of Gauss points reduces considerably the number of required time steps, Fig. 10-(b).

\subsection{Minimum relative permeability}

Convergence of the time marching scheme requires the relative permeability to be given a non zero value: the relative permeability features in the diffusion coefficient (15) and therefore also in the diffusion terms of the tangent matrix (27). A modification of the constitutive equations that define the relative permeabilities was adopted to ensure 


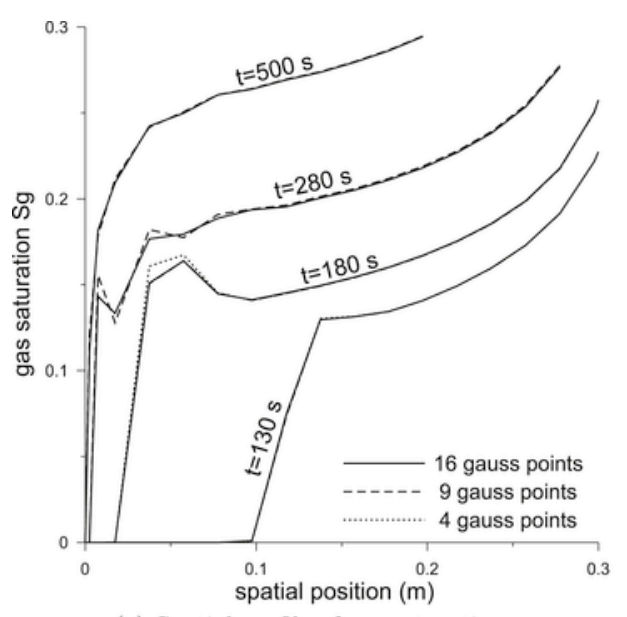

(a) Spatial profile of gas saturation

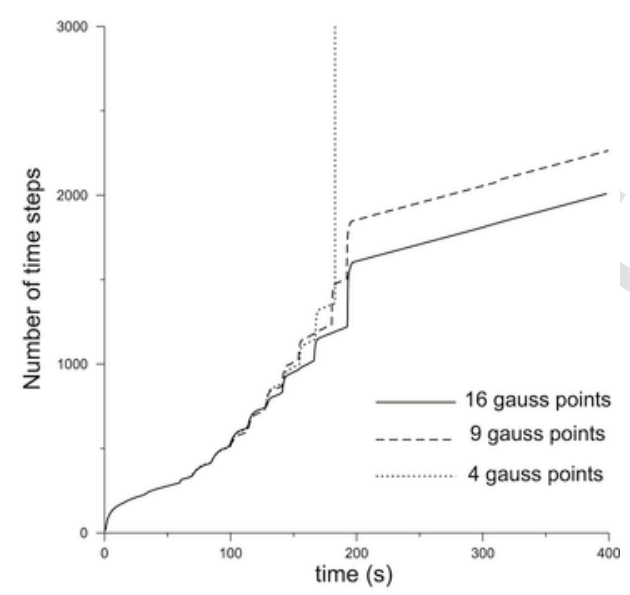

(b) Total step number

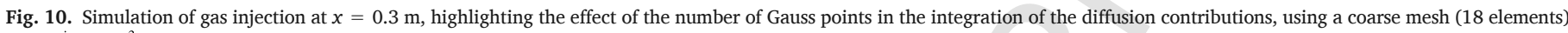

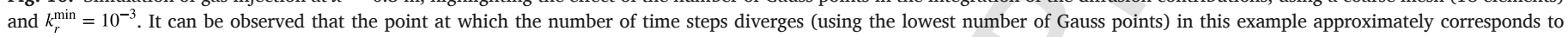
$\mathrm{t}=180$ s. Saturation profiles are not comparable with previous plots, as they were obtained with different initial conditions $\left(S_{\mathrm{W}}=0.3, S_{\mathrm{O}}=0.7\right)$ and boundary conditions.

a minimum relative permeability. The reference value $k_{r}^{\min }=10^{-4}$ was observed to provide meaningful outputs. A reduction by one order of magnitude of $k_{r}^{\mathrm{min}}$ slightly increases the propagation speed of the saturation front, Fig. 11. When the saturation is smaller than about 0.28 for water and 0.075 for gas, the resulting relative permeability is, according to eqn. (A.12), of the order of $10^{-3}$. In fact below such saturation thresholds, the relative permeability obtained with the Brooks-Corey model is negligible. Therefore the resulting water permeability is about $10^{-3}$ over most of the core but in the vicinity of the outlet, cf. Fig. 6. Thus, the water pressure profiles are essentially linear over the corresponding part of the core.

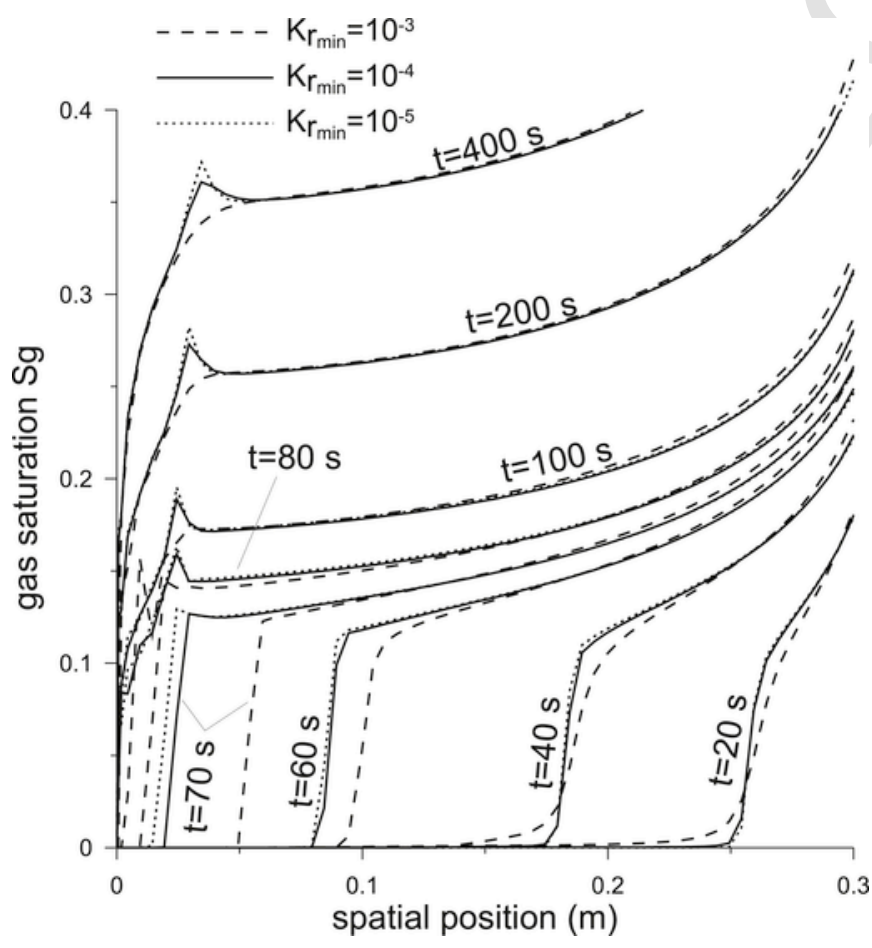

Fig. 11. Simulation of gas injection at $x=0.3 \mathrm{~m}$. Effects of the minimum relative permeability on the gas saturation.

\subsection{Deviation from the consistent Newton's iterative procedure}

The semi-discrete nonlinear equations are solved through a full Newton-Raphson scheme. However, at certain stages of the simulation process, the iterative scheme could not converge, i.e. it required a definitely too small time step. To address this issue, two temporary modifications of the tangent matrix have been adopted. These modifications, and the on/off switch criteria to activate them, are briefly described hereafter.

The storage coefficients $d_{k j}$ defined by (29) stem from the linearization of the storage term, eqn (22). They feature in the tangent matrix through the element matrices $\mathbf{D}_{k j}^{e}$, eqns (30) and (33). The actual critical stage for the gas injection process occurs when the gas front reaches the outlet, where the pressure gradients of oil and gas are large. The contribution to the tangent matrix due to these gradients is explicit in (27). It can be large close to gas breakthrough so as to make the diagonal terms of the diffusion matrix very small or even negative and the capillary pressure $p_{g}-p_{0}$ negative. Since then the gas saturation is small, the derivatives with respect to pressures detailed in Appendix A may become inaccurate. Usually, at this point the number of iterations of the full Newton-Raphson scheme tends to increase, thus the time steps become very small. The scheme is then modified as follows: a) if, after $7 \mathrm{~N}$ iterations, convergence is not achieved, the storage coefficient $d_{g g}$ is set to $c_{\text {min }}=n \rho_{\mathrm{g}} \max \left(S_{\mathrm{g}}+K_{\mathrm{g}} \partial S_{\mathrm{g}} / \partial p_{\mathrm{g}}, 0.1\right) / K_{\mathrm{g}}$ (with $K_{\mathrm{g}}$ the bulk modulus of gas phase) and b) if the capillary pressure $p_{\mathrm{g}}-p_{j}$ is negative, the derivatives $\partial D_{\mathrm{g}} / \partial p_{j}$ in the diffusion contribution (27) $)^{1}$ and $\partial S_{\mathrm{g}} / \partial p_{j}$ in the storage contribution $d_{g j}$, eqn (29), are set to zero. Let us remark that the switch to this modification is decided at each step. In fact, while the modified scheme appears more robust than the full Newton-Raphson scheme, it slows down considerably the convergence process if it is adopted systematically. This feature is illustrated in Fig. 12, where the effect of adopting the modified scheme on the number of time steps required during the gas injection simulation is explored. It turns out that the modified scheme is much more efficient than full Newton-Raphson before gas breakthrough, but it does not need to be activated at later times. 


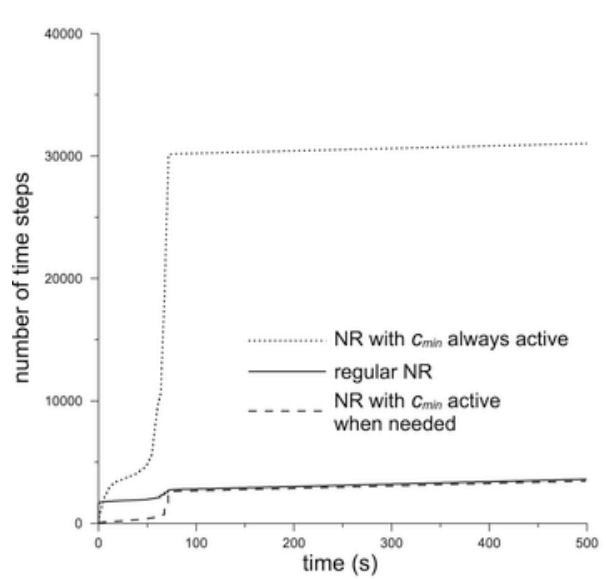

(a) Total step number

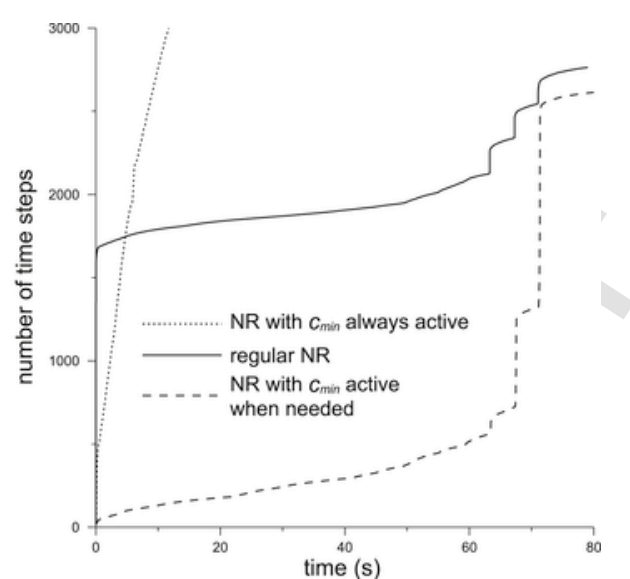

(b) Blow up of left plot

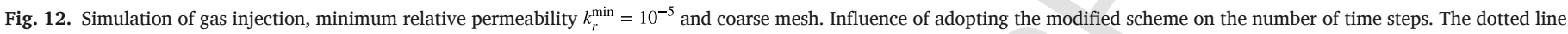

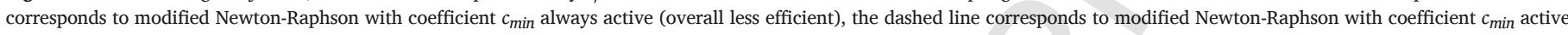
only when needed (overall more efficient) and the solid line corresponds to regular Newton-Raphson. Plot (b) is an enlargement of plot (a) at early times, until gas breakthrough.

\subsection{Time step selection}

The time step selection which aims at keeping the number of iterations between four and seven uses the following scheme:

- if the number of iterations becomes greater than seven or if the line search coefficient is smaller than $10^{-3}$, then the step is resumed and a new trial is performed with a time step decreased of $30 \%$;

- at the end of a time step:

- if just 1 trial was needed with less that 4 iterations in the last time step, then the time step is increased by $20 \%$;

- if the number of trials was greater than 1 , the time step is decreased by $20 \%$.

In addition, the step is bounded below and above, namely $10^{-5} \mathrm{~s} \leq \Delta t \leq 0.62 \mathrm{~s}$. Typically, the step is observed to decrease continuously as gas breakthrough is approached, and to subsequently jump to the given upper bound (Fig. 13).

\subsection{Convergence criteria}

The convergence criteria are based on the absolute differences between two iterations of both unknowns and residuals. The difference between displacement vectors should be smaller than $10^{-5} \mathrm{~m}$ and the difference between pressures should be smaller than $0.1 \mathrm{~Pa}$. The out of balances of momentum and fluid mass are each checked to be smaller than $0.5 \times 10^{-3}$ times their values at iteration 1 .

\subsection{Influence of mesh distortion}

The effects induced by mesh distortion are examined on the reference fine mesh, consisting of 63 elements having a base width of $2 \mathrm{~cm}$ and a height of $0.5 \mathrm{~cm}$ (along sample axis), described in Sect. 6.1 and shown in Fig. 9. As previously stated, the FE mesh is refined close to the outflow boundary, with a maximum element enhancement of 1/8.

In the central part of the sample, the finite elements are progressively distorted through skewing, starting from the inflow and outflow boundaries (where the distortion is null), through to the mid point of the sample (where the distortion is maximum). Fig. 14 shows the typical element distortion at various locations along the sample.
The space profiles of gas saturations computed along the two (upper and lower) sides of the sample are also shown in Fig. 14. Clearly, mesh distortion introduces only a small difference between the saturations along the two sides of the element. In fact, if mesh distortion had no effect at all, the dashed and continuous curves of Fig. 14 would perfectly overlap.

\section{Co- and counter-current imbibition tests}

In imbibition tests, the wetting phase (water for a water wet rock) displaces the non wetting phases (oil and gas). In co-current imbibition tests, all three phases move in the same direction. In three phase counter-current imbibition tests, oil and gas are pushed out of the core over the same boundary through which water is forced in.

Two fluid counter-current imbibition onto mixed wet rocks saturated with water and oil has been addressed in a number of publications, e.g. (Behbahani and Blunt, 2005). Gravity effects are considered in the co-current imbibition tests of El-Amin and Sun (2011) where the water flux and the oil pressure are set to vanish at the outlet. Here, in contrast, the specimen is horizontal, the rock is water wet and it is saturated by three fluids, namely water, oil and gas.

Spontaneous imbibition is simulated by decreasing the capillary pressures $p_{\mathrm{o}}-p_{\mathrm{w}}$ and $p_{\mathrm{g}}-p_{\mathrm{o}}$ to zero. For that purpose, the saturations are driven to the water vertex I (in Fig. 1), where the effective saturation of water is equal to 1 and therefore the effective saturations of oil and gas vanish. While, in the simulations, the primary variables, namely the pressures, are manipulated to ensure a linear variation of the saturations with time, other controls to target the water vertex at the inlet are possible.

The imbibition tests presented here, aimed at studying enhanced oil recovery, consider water, oil and air as the three saturating fluids. However, this work is also relevant to other important industrial challenges involving three immiscible fluids, such as air sparging for soil decontamination and geological sequestration of carbon dioxide in coal seams.

\subsection{Initial and boundary conditions}

The initial saturation conditions, shown in Fig. 15, are uniform over the deformable core of length $L=0.3 \mathrm{~m}$. The hydraulic boundary conditions at the ends of the core are sketched in Fig. 16. As for the mechanical boundary conditions, the lateral displacement is set to vanish all along the core, while the axial displacement at $x=L$ is set to zero. 


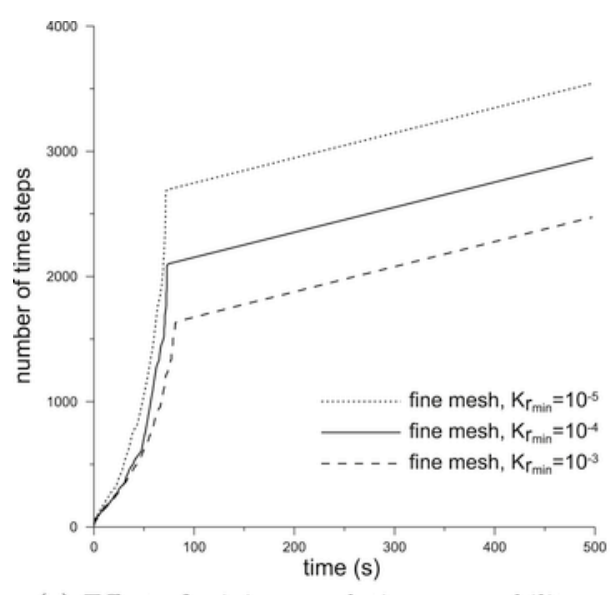

(a) Effect of minimum relative permeability

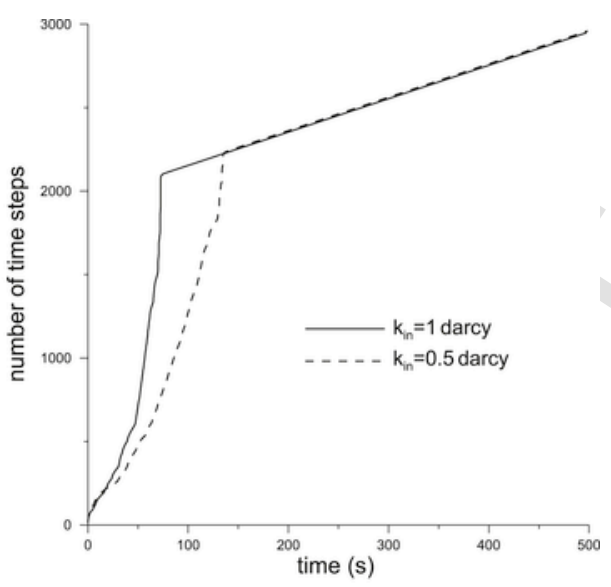

(b) Effect of absolute permeability

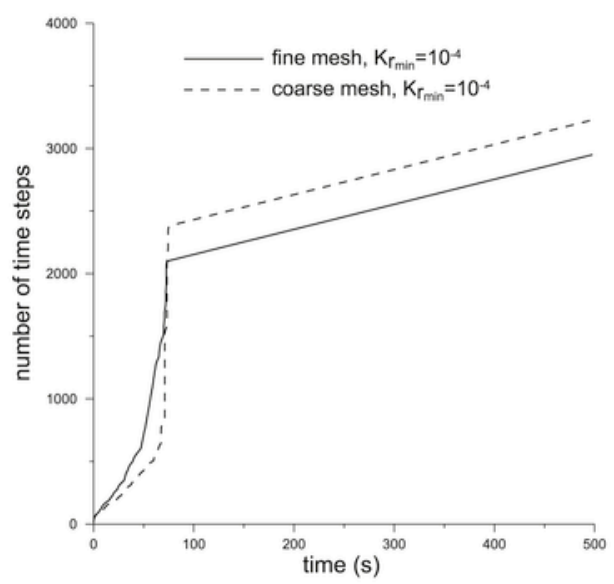

(c) Effect of mesh size

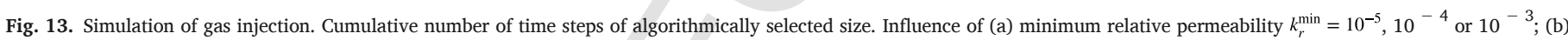

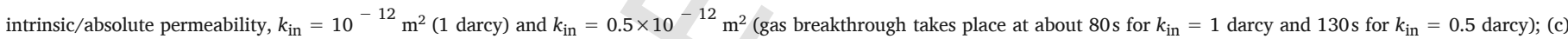
mesh size.

At the inlet $x=0$, the total traction is kept to its initial value, $\sigma_{x x}(x=0, t)=-\kappa \bar{p}(x=0, t=0)$.

In co-current imbibition, the normal mass flux of oil $j_{\mathrm{o}}=-D_{o} \nabla p_{0} \cdot \mathbf{n}$ at the right end $x=L$, where $D_{o}$ [ unit: $\mathrm{s}$ ] is the diffusion coefficient defined by (15), is controlled by a special convective boundary condition, i.e.

$j_{\mathrm{o}}=k_{\mathrm{o}, \mathrm{c}}\left(p_{\mathrm{o}}-p_{o, e x t}\right)$,

where $p_{o, \text { ext }}=p_{\mathrm{g}}(x=L, t)=0$. The boundary condition is numerically designed so as to obtain a smooth transition between an initial Neumann-type condition (no flux) and an eventual Dirichlet-type condition (fixed pressure). In other words, if the convective coefficient $k_{\mathrm{o}, c}$ [ unit:

$\mathrm{s} / \mathrm{m}$ ] is large, continuity of the oil pressure at the boundary is recovered while, on the other hand, if $k_{\mathrm{o}, c}$ is zero the normal oil mass flux vanishes. In fact in the co-current imbibition problem, oil flux should vanish initially, because $p_{\mathrm{o}}-p_{o, \text { ext }}<0$, while $p_{\mathrm{o}}-p_{o \text {,ext }}>0$ is prevented at later times.

To obtain the above described transition, a pressure differentiable expression is proposed providing a gradual variation of $k_{\mathrm{o}, c}$ from zero (at $p_{\mathrm{o}}-p_{o, \text { ext }}<0$ ) to a large value (for $p_{\mathrm{o}}-p_{o, \text { ext }}>1$ ), over the interval $0<\left(p_{0}-p_{o, \text { ext }}\right)<1 \mathrm{kPa}$, as follows: $k_{\mathrm{o}, c}=10^{3} \times \frac{\rho_{\mathrm{o}}}{L_{e}} \frac{k_{\mathrm{in}}}{\eta_{\mathrm{o}}} \times \sin ^{2}\left(\frac{\pi}{2} \tilde{\alpha}\right)$.

where $L_{e}$ is the element size close to the boundary, and $\tilde{\alpha}=\min (\max (\alpha, 0), 1)$, with $\alpha \equiv\left(p_{\mathrm{o}}-p_{o, \text { ext }}\right) / \Delta$ and $\Delta=1 \mathrm{kPa}$.

The adopted transport and mechanical constitutive parameters are the same as those listed in Tables 2 and 3 respectively, but the irreducible saturations shown in Table 4 are now used. A uniform, fine mesh with 60 elements and a minimum relative permeability $k_{r}^{\min }=10^{-3}$ are henceforth adopted as standard. The diffusion contributions of both the residual and the tangent matrix are integrated through $2 \times 2$ Gauss points. Also in this case, the mass supplies are set to zero.

\subsection{Three-fluid phase permeability models}

The two phase permeability curves obtained with the Brooks-Corey model are symmetric at large values of exponent $\lambda$ only. An alternative permeability model, that we may refer to as symmetric formulation, does not differentiate the wetting and non wetting fluids, as follows

$k_{r \mathrm{w}}=\left(\underline{S}_{\mathrm{w}}\right)^{\omega}, \quad k_{r \mathrm{o}}=\left(\underline{S}_{\mathrm{o}}\right)^{\omega}, \quad k_{r \mathrm{~g}}=\left(\underline{S}_{\mathrm{g}}\right)^{\omega}$. 


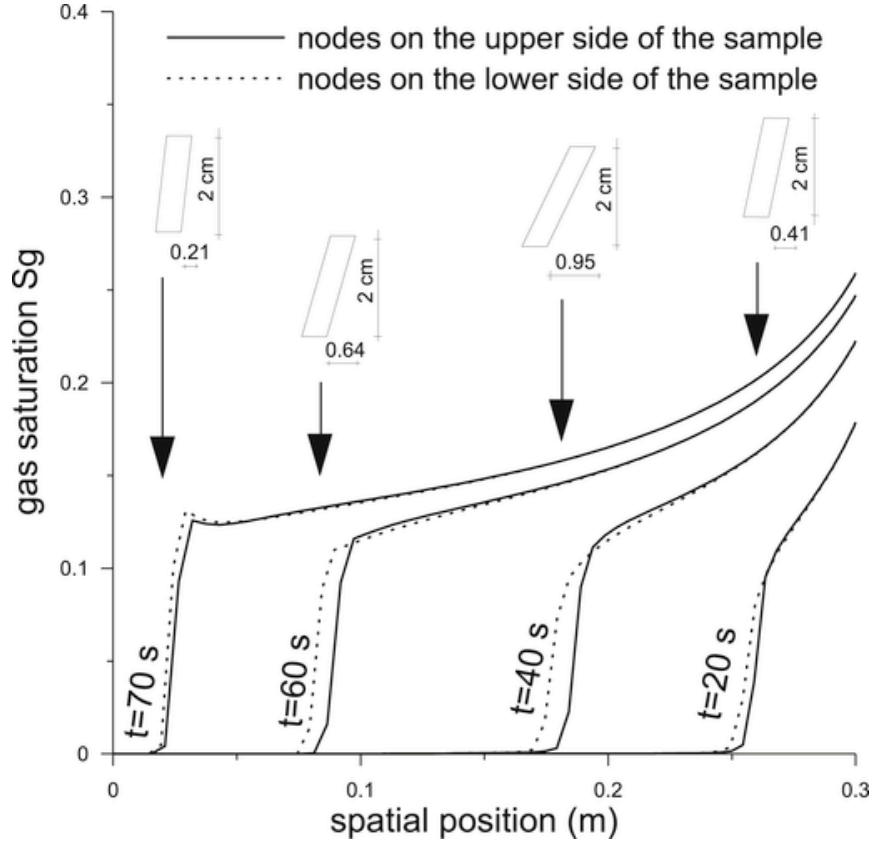

Fig. 14. Simulation of gas injection at $x=0.3 \mathrm{~m}$. Influence of mesh distortion.

$$
\begin{aligned}
& \mathrm{S}_{\mathrm{w}}=0.110 ; \mathrm{p}_{\mathrm{w} 0}=\mathrm{p}_{\mathrm{g} 0}-\mathrm{p}_{\text {cgw }}\left(\mathrm{S}_{\mathrm{w}}, \mathrm{S}_{0}, \mathrm{~S}_{\mathrm{g}}\right)=-1610.6 \mathrm{kPa} \\
& \mathrm{S}_{0}=0.512 ; \mathrm{p}_{\mathrm{o} 0}=\mathrm{p}_{\mathrm{g} 0}-\mathrm{p}_{\text {cgo }}\left(\mathrm{S}_{\mathrm{w}}, \mathrm{S}_{0}, \mathrm{~S}_{\mathrm{g}}\right)=-19.9 \mathrm{kPa} \\
& \mathrm{S}_{\mathrm{g}}=0.378 ; \mathrm{p}_{\mathrm{g} 0}=0 \mathrm{kPa} \\
& \mathrm{x}=0 \quad \mathrm{x}=\mathrm{L}=0.3 \mathrm{~m}
\end{aligned}
$$

Fig. 15. Initial conditions for a 1D imbibition test. The saturations are prescribed uniform over the core. The pressures and capillary pressures are deduced from the saturations based on the constitutive relations. The initial fluid volume fraction is equal to $n=0.4$.

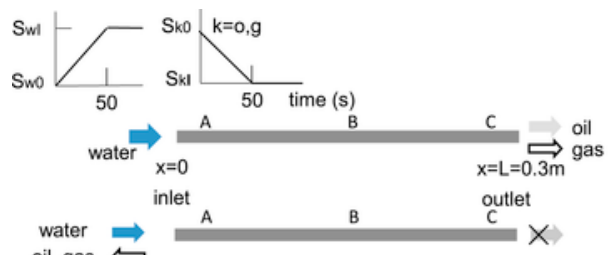

co-current imbibition :
$\mathrm{pg}=0, \mathrm{jw}=0$
$\mathrm{jo}$ convective bc
counter-current imbibition
$\mathrm{jw}=\mathrm{jo}=\mathrm{jg}=0$

Fig. 16. Boundary conditions for a $1 \mathrm{D}$ imbibition test into a core which is initially almost completely saturated by oil and gas. At the left end $x=0$, the gas pressure is maintained to $0 \mathrm{kPa}$, the oil and gas saturations are brought to their irreducible values (point I in Fig. 1) in 50 s. Using relations (A.1)-(A.6), linear rates are achieved by manipulating the water and oil pressures which end up to $0 \mathrm{kPa}$. For co-current imbibition, at the right end ( $x=L$ ), the gas pressure is maintained to zero, the water flux $j_{\mathrm{W}}$ vanishes, water being more wetting than gas and oil, and the oil flux $j_{\mathrm{o}}$ is controlled through a convective boundary condition. For counter-current imbibition, all fluxes $j_{k}, k=\mathrm{w}, \mathrm{o}, \mathrm{g}$ at $x=L$, are set to vanish.

Table 4

Irreducible saturations (Hustad, 2000). Note that $S_{i r j}$ denotes the irreducible saturation of fluid $i$ in the presence of the sole fluid $j$.

$\begin{array}{ccc}\text { water-oil } & S_{\text {wro }}=0.15 & S_{\text {orw }}=0.2 \\ \text { oil-gas } & S_{\text {org }}=0.04 & S_{\text {gro }}=0.03 \\ \text { gas-water } & S_{\text {grw }}=0.06 & S_{\text {wrg }}=0.05\end{array}$

In the above, for an exponent $\omega=1$, the relative permeabilities exhibit linear variation in terms of effective saturations. On the other hand, a large exponent $\omega$ causes the relative permeability curves to become more 'L-shaped' and gives rise to steep propagation fronts.

A further alternative is the so-called Stone model (Bell et al., 1986), formulated as

$$
\begin{array}{r}
k_{r \mathrm{w}}=1.09\left(\underline{S}_{\mathrm{w}}\right)^{1.516687}-0.09\left(\underline{S}_{\mathrm{r}}\right. \\
k_{r \mathrm{o}}=\left(1.19\left(1-\underline{S}_{\mathrm{g}}\right)^{1.006}-0.19\left(1-\underline{S}_{\mathrm{g}}\right)^{1.024}\right)(1.95(1- \\
k_{r \mathrm{~g}}=0.525\left(\underline{S}_{\mathrm{g}}\right)^{1.02}+0.475(\underline{S}
\end{array}
$$

The relative permeability curves along the edges of the irreducible saturation triangle are all 'L-shaped' (with upward concavity) for $\omega>1$ . In a similar fashion to the Brooks-Corey model, the contours of the relative permeabilities $k_{r \mathrm{w}}$ and $k_{r g}$ of these alternative models are parallel to the respective oil-gas and water-gas edges of the irreducible saturation triangle. The contours of the relative permeability to oil $k_{r o}$ are concave with respect to the oil vertex for all models, except for the symmetric formulation with $\omega=1$. The above described relative permeability models have been employed as alternatives to the Brooks-Corey model in the simulations; however, with the main aim of testing their overall numerical performance, no accurate parameter calibration on specific experimental datasets was attempted.

Further, the regime of the field equations obtained with the above models was checked along the lines of the analysis of (Bell et al., 1986) and (Juanes and Patzek, 2004). When the capillary forces are neglected along (Bell et al., 1986), the sole model which displays a finite zone where hyperbolicity is lost is the Stone model. For the other models, hyperbolicity holds strictly except possibly at certain isolated points where two wave-speeds are equal (non strict hyperbolicity).

Therefore one may consider that the computational difficulties encountered in the simulations of gas injection reported in Sect. 5, using the numerical scheme described in Sect. 4, are not directly linked to the regime of the partial differential equations. Moreover, gas injection has been observed in (Gajo et al., 2017) to give rise to the propagation of a gas saturation front at finite speed for all models but the Stone model and the symmetric formulation with $\omega=1$, which showed a definitely faster front. While a finite zone where hyperbolicity is lost is observed for the Stone model, this is not the case for the symmetric formulation with $\omega=1$. Hence the faster propagation observed for these two models, for gas injection as well as for the imbibition tests described below, cannot be ascribed to the regime of the field equations.

\subsection{Simulation tests}

Fig. 17 shows the space profiles at different time instants of the pressures and saturations for co-current imbibition simulations using the Brooks-Corey relative permeability model. In addition to this model, simulations were carried out using the Stone model and the symmetric formulation both with $\omega=1$ and $\omega=1.5$. However, the corresponding figures are not shown here for the sake of brevity.

Water imbibition causes a steep propagation front for the gas saturation (Fig. 17-(b)), while the propagation fronts of water and oil saturation (Fig. 17-(d) and -(f), respectively) have smaller amplitudes. Indeed, for each two phase system, the so-called mobility ratio can be expressed as the ratio of the viscosity of the displaced fluid over the viscosity of the displacing fluid. The mobility ratio is equal to 2 for the water-oil system and to 0.01 for the water-gas system, and is known to be an indicator of the ease of sweep. On the other hand, while the irreducible saturations are fixed in this analysis, pore scale simulations in- 

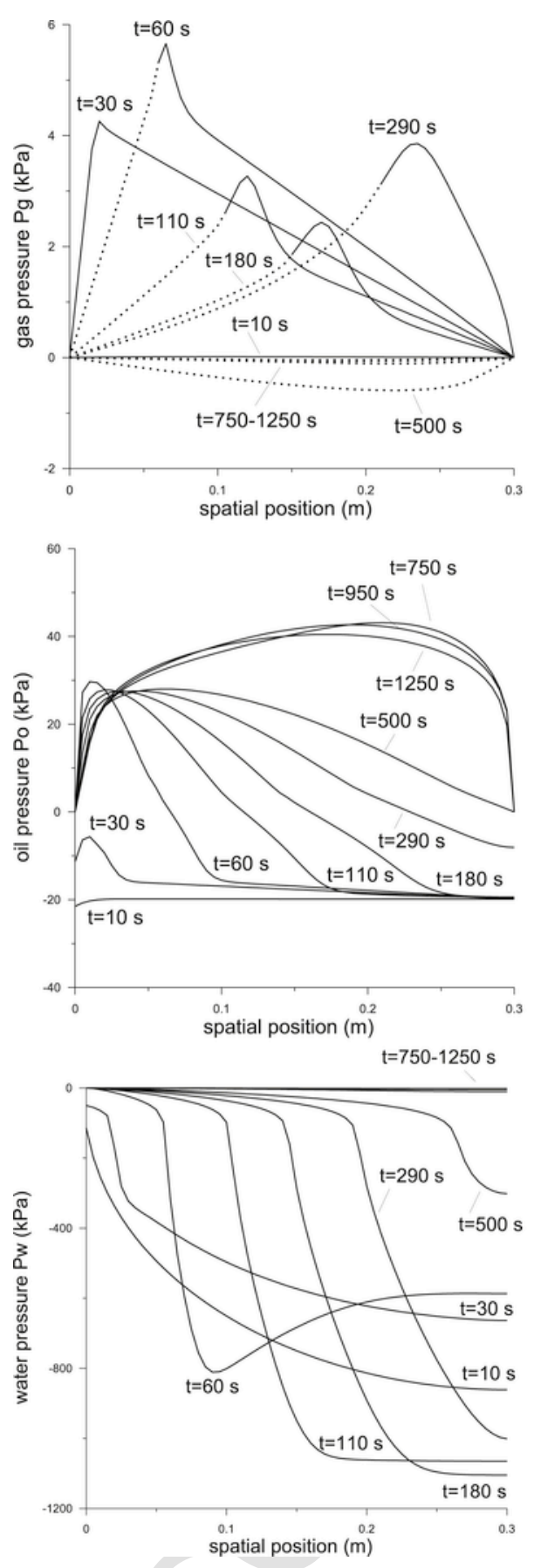
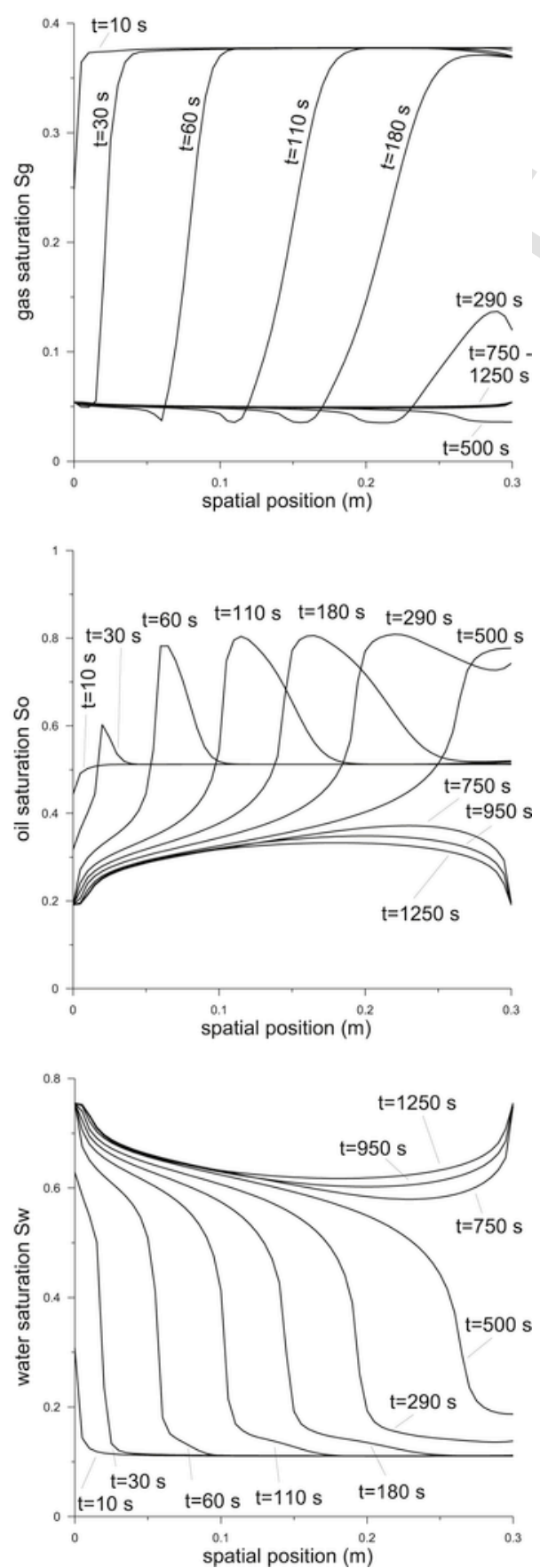

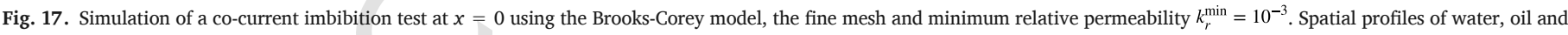
gas pressures and saturations at various times. The dotted curves correspond to a vanishing gas saturation.

dicate that gas invasion induce piston-like displacements of the wetting phases and results in larger oil recovery than water injection that leads to snap-off displacements in water wet rocks, disconnecting oil clusters (Al-Dhahli et al., 2013).

Gas is pushed out of the core at a much faster rate than oil. In fact, gas pressure is maintained to zero at the right end and its saturation is kept to its irreducible value at the left end, so that gas is evacuated quickly in favor of oil. The time needed for the gas front to reach the right end ranges from 100 s to 200 s depending on the relative permeability model. These figures agree with the characteristic time for water-wet systems ((Ma et al., 1997), (Gajo et al., 2017)).
As gas is expelled from the specimen, there is a temporary burst of gas pressure for all tested models at about $150 \mathrm{~s}$, with special regards to the Stone model and the symmetric formulation with $\omega=1.5$ (the corresponding figures are not shown for brevity). This point can be understood by looking at the saturation path in the ternary diagram, Fig. 19-(b). Since gas is expelled, the saturation path tends toward the oil-water edge (at later times, it will tend to the water vertex). Along this path, the capillary pressure $p_{\mathrm{g}}-p_{\mathrm{w}}$ passes through a maximum as can be realized by considering Fig. 3 . The capillary pressure burst is more pronounced for points $\mathrm{B}(x=0.16 \mathrm{~m})$ and $\mathrm{C}(x=0.29 \mathrm{~m})$ closer to the right end for which the saturation path comes closer to the oil vertex, where the capillary pressure $p_{\mathrm{g}}-p_{\mathrm{w}}$ is larger. 
Once the water front has reached the right end, its saturation increases there, since water outflow is prevented. Moreover, since gas has been expelled quickly, the saturation is close to the oil-water edge where the capillary pressure $p_{\mathrm{g}}-p_{\mathrm{o}}$ vanishes, Fig. 3 . In the long range, the saturation path should actually tend to the water vertex where the three pressures are equal. The gas pressure being kept to zero at the right end, the water and oil pressure should also tend to this value. Meanwhile, oil has been pushed to the right end but it has not yet been flushed out of the core, and it is somehow trapped in the middle of the core, due to the rise of the water saturation at the right end. In fact, while water has not been able to push oil from the left end as easily as gas, it finally succeeds in sucking oil out of the specimen. Indeed, since water is more wetting than oil, and water pressure increases at the right end due to gas expulsion as explained above, oil pressure is forced to decrease, not only in the vicinity of the left end, but also close to the right end. Oil saturation and pressure display non monotonous spatial profiles which are slow to disappear.

Gas propagation displays a front for all models, including the Stone model and the symmetric formulation with $\omega=1$, but the propagation time scales for these two models are about half of the time scales associated with the Brooks-Corey model. Furthermore, using the Stone model, water was observed to be unable to push oil out of the core center, as the oil saturation profiles exhibited substantially different patterns to those shown in Fig. 17. Anyway, the gas saturation profiles obtained with the Stone model were very similar to those obtained with Brooks-Corey. In contrast, water was able to push oil out for the symmetric formulation with $\omega=1$.

For the counter-current imbibition simulations shown in Fig. 18, oil cannot exit the core at the right end. However, as oil has been pushed to that end, its saturation and pressure in that zone increase for a certain period, before it can escape to the left end where its saturation is maintained to its irreducible value.

In all simulations discussed above, the total traction applied at the left end is maintained to its initial value. The effective stress $\sigma_{x x}{ }^{\prime}(x=0, t)=\kappa(\bar{p}(x=0, t)-\bar{p}(x=0, t=0))$, which is initially zero, becomes positive (tensile) when the saturations of oil and gas are reduced to their irreducible values as the average pressure increases all along the core, Fig. 17. In fact the fluid pressures were initially negative (or zero for gas) and they end up to zero. As a consequence of this increase in average pressure at constant total traction, the core undergoes an extension at late times. The right end being fixed, the displacement takes place toward the free left end, as shown in Fig. 19-(a) and 18-(a).

The space and time evolutions of the pressures result in a continuous extension of the core for the Brooks-Corey model. Using the other models, the water pressure reaches very negative values at early times, resulting in temporary contraction of at least part of the core (plots are not shown for the sake of brevity).

By comparing the core displacement (extension) for co-current imbibition (Fig. 19-(a)) and counter-current imbibition (Fig. 20-(a)), no major differences are observed, apart from a more homogeneous and progressive rate of displacement for the latter case. The less uniform displacement occurring at early times during co-current imbibition is due to the evolution of the average fluid pressure $\bar{p}$. On the other hand, the saturation path for counter-current imbibition (Fig. 20-(b)) is rather different from its co-current imbibition counterpart (Fig. 19-(b)). In counter-current imbibition, only at point $C(x=0.28 \mathrm{~m})$ does the path approach the oil vertex, whereas at points $\mathrm{B}(x=0.16 \mathrm{~m})$ and $\mathrm{A}$ $(x=0.02 \mathrm{~m})$ the saturation path moves gradually towards the water apex. This aspect is also reflected by the gas saturation profiles for the two test types (Fig. 17-(b) and 18-(b)).

The standard value of minimum relative permeability has been fixed to $10^{-3}$, although lower values have also been used. A low value is expected to slow down somehow the diffusion process. However, the effect in the spatial profiles of oil saturation was found to be rather small.

\section{Conclusion and future developments}

A comprehensive numerical scheme to simulate the diffusion of three immiscible fluid phases in a deformable porous solid was developed within a FE framework. It is based on the individual mass balance of each phase, without resorting to simplifications, including consistent lumping of the storage terms and without any linearization in the expression of the residual.

The proposed computational framework was shown to be robust and flexible, and to correctly reproduce the response of rock samples in standard laboratory testing configurations. Consistent results were obtained for gas injection simulations describing drainage in a water wet deformable core. The influence on results of changing initial saturations and rock's intrinsic permeability is also explored. Co- and counter-current imbibition tests were also simulated, exploring the impact of adopting different permeability models, bringing about some differences in the timescale of front propagation and in the saturation paths.

Further, the sensitivity of results to some key computational ingredients was assessed. Coarsening the mesh induces oscillations in the propagation front, although it does not affect its speed of propagation. Increasing the number of Gauss points does not affect the results significantly but considerably reduces the number of time steps. Convergence of the time marching scheme was found to require a minimum non zero relative permeability. Transient departures from the standard Newton-Raphson scheme were also found to be beneficial in the vicinity of gas breakthrough.

Further developments of this framework include:

(1) testing the so-called passive air phase assumption as a particular case of the fully three-phase modelling scheme. While the number of primary variables is reduced by one, the constitutive equations for relative permeabilities and capillary pressures still need to consider the three immiscible fluids;

(2) a more systematic numerical study of testing paths where saturation cyclically switches from a two phase to a three-phase situation, with applications in real situations where the saturation domains evolve with time, requiring the position of the saturation interfaces to be tracked. While this aspect is already covered here, it deserves a more systematic analysis;

(3) consideration of thermal effects and of phase changes affecting water, i.e. vaporisation and steam condensation, relevant to modelling the SAGD technique;

(4) implementing a thermo-mechanical elasto-plastic model, based on the Cam-Clay family, to properly account for the thermo-hydro-mechanical couplings involved in three-phase flow problems, with particular reference to SAGD;

(5) extending this framework to mixed-wet and oil-wet rocks.

\section{Acknowledgements}

A. Gajo acknowledges support from the EU research project ERC-2013-ADG-340561-INSTABILITIES. F. Cecinato acknowledges support from the EU research project PIAPPGA-2013-609758-HOTBRICKS. B. Loret acknowledges support from the "Visiting Professor 2013" program of the University of Trento.

\section{Appendix A.}



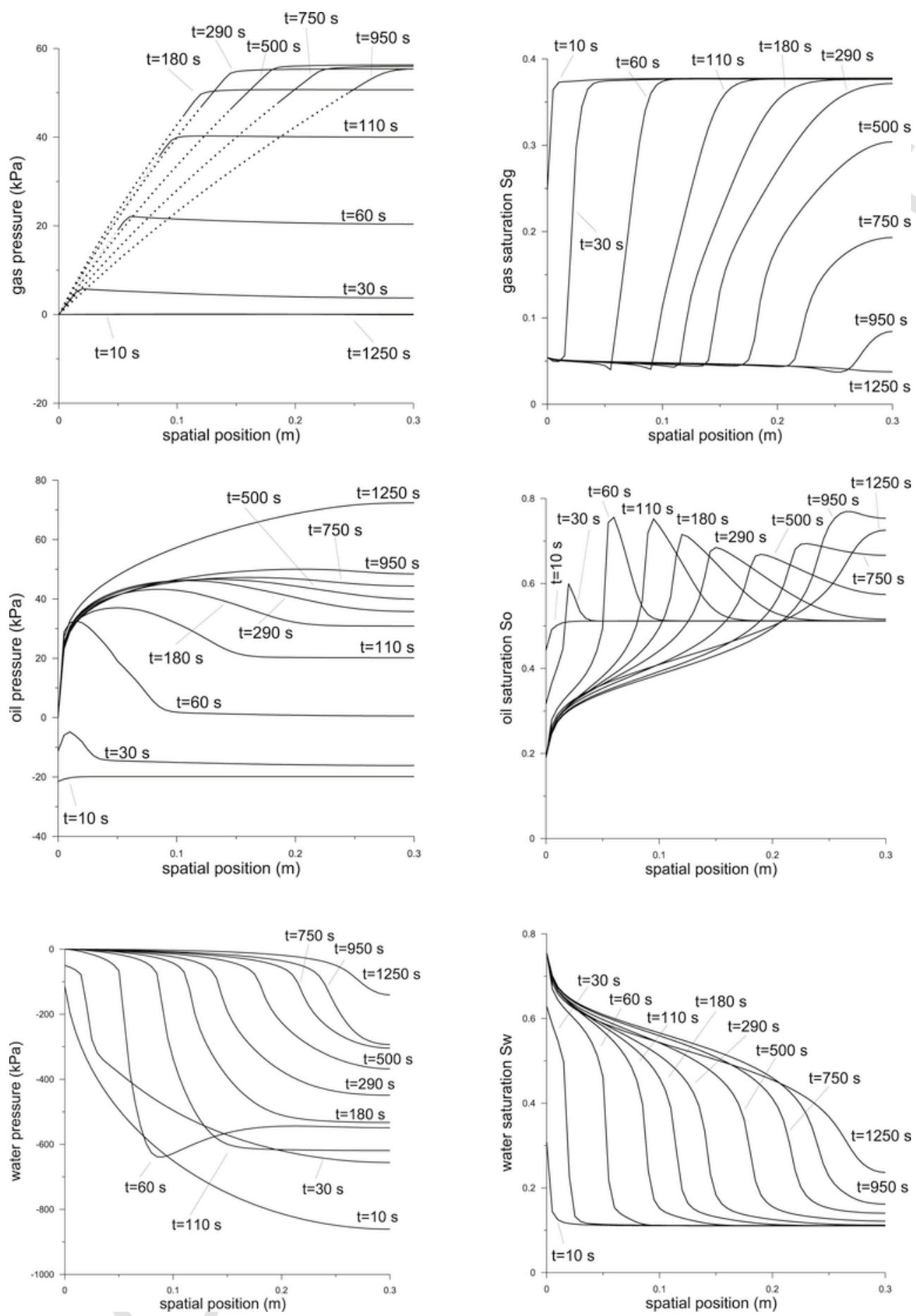

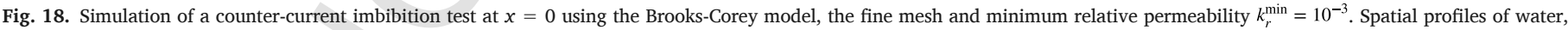
oil and gas pressures and saturations at various times. The dotted curves correspond to a vanishing gas saturation.

It is remarked that the two effective saturations $\left(\underline{S}_{\mathrm{w}}, \underline{S}_{\mathrm{o}}\right)$ are considered as independent variables, implying the replacement of $\underline{S}_{\mathrm{g}}$ with $1-\underline{S}_{\mathrm{w}}-\underline{S}_{\mathrm{o}}$.

\section{A.1. derivatives of saturation with respect to pressures}

The derivatives $\underline{\mathbf{X}}=\left[\underline{X}_{i j}\right]=\left[d \underline{S}_{i} / d p_{j}\right]$ are obtained as follows. 


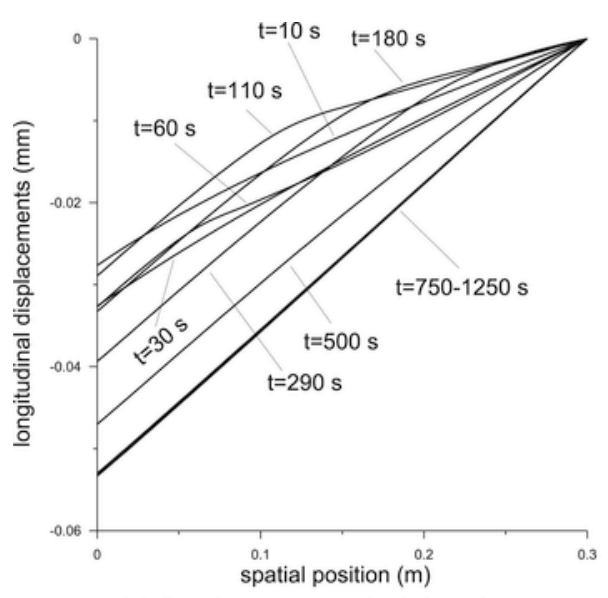

(a) Displacement at the left end

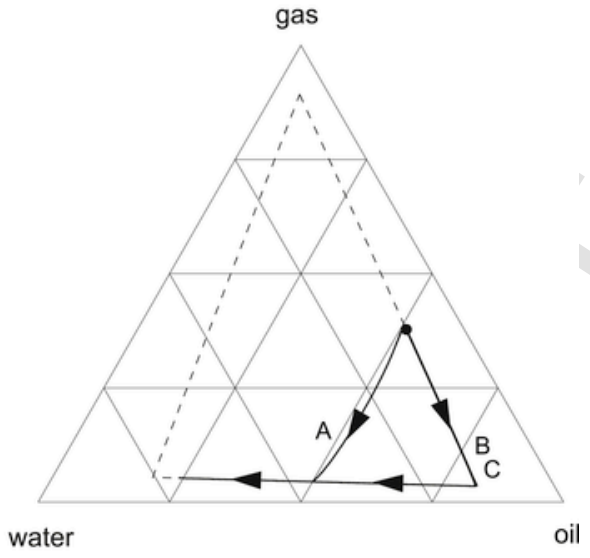

(b) Saturation path

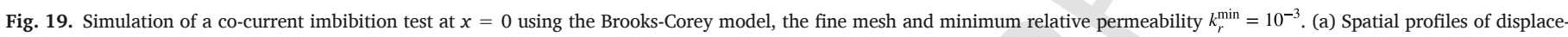

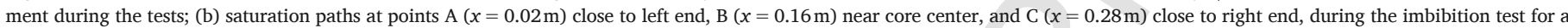

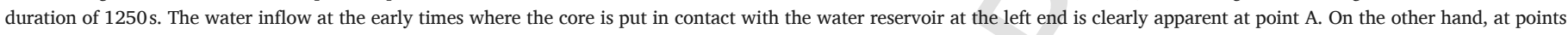
$\mathrm{B}$ and $\mathrm{C}$, gas is first expelled through the right end and the water front needs a significant time to reach these points.

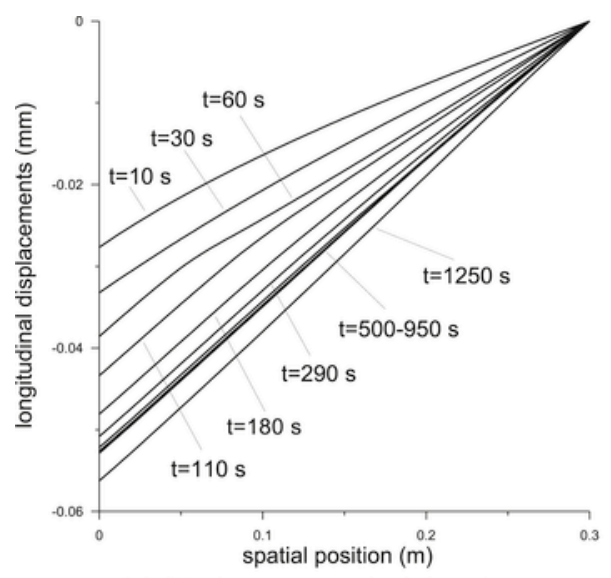

(a) Displacement at the left end

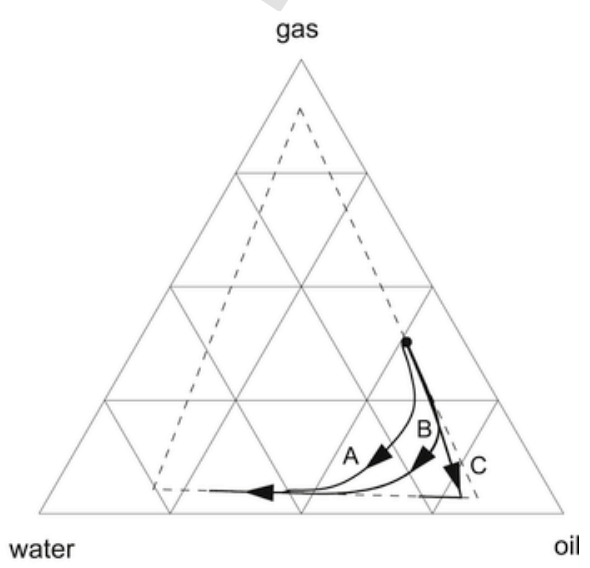

(b) Saturation path

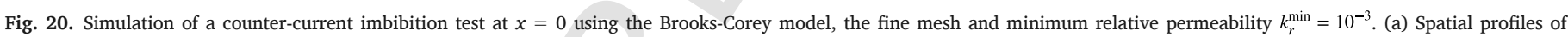

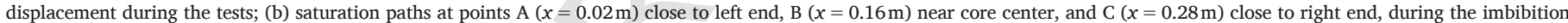

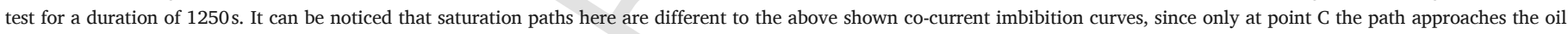
vertex, whereas at points B and A the saturation path moves gradually towards the water apex.

The starting point is the knowledge of two capillary relations,

$$
\begin{aligned}
p_{\mathrm{o}}-p_{\mathrm{w}} & =p_{\text {cow }}\left(\underline{S}_{\mathrm{w}}, \underline{S}_{\mathrm{o}}\right) ; \quad p_{\mathrm{g}}-p_{\mathrm{o}} \\
& =p_{\text {cgo }}\left(\underline{S}_{\mathrm{w}}, \underline{S}_{\mathrm{o}}\right) .
\end{aligned}
$$

The above relations are differentiated,

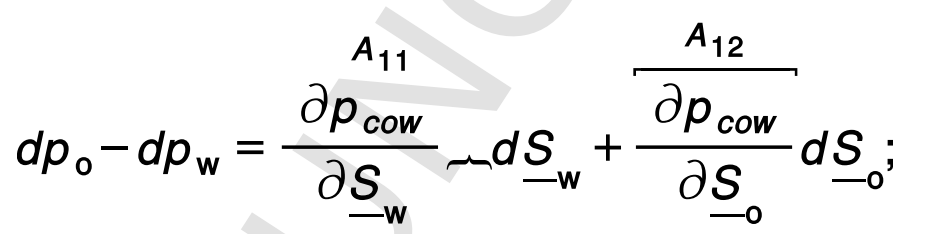

and the result is inverted,

$$
\begin{aligned}
\operatorname{det} & \mathbf{A}\left[\begin{array}{l}
d \underline{S}_{\mathrm{w}} \\
d \underline{S}_{\mathrm{o}}
\end{array}\right] \\
= & {\left[\begin{array}{cc}
A_{22} & -A_{12} \\
-A_{21} & A_{11}
\end{array}\right]\left[\begin{array}{l}
d p_{\mathrm{o}}-d p_{\mathrm{w}} \\
d p_{\mathrm{g}}-d p_{\mathrm{o}}
\end{array}\right] } \\
= & {\left[\begin{array}{c}
-A_{22} d p_{\mathrm{w}}+\left(A_{12}+A_{22}\right) d p_{\mathrm{o}}-A_{12} d p_{\mathrm{g}} \\
A_{21} d p_{\mathrm{w}}-\left(A_{11}+A_{21}\right) d p_{\mathrm{o}}+A_{11} d p_{\mathrm{g}}
\end{array}\right], }
\end{aligned}
$$

with $\operatorname{det} \mathbf{A}=A_{11} A_{22}-A_{12} A_{21}$. Hence the derivatives of the effective saturations, namely for $i=w, o, j=\mathrm{w}, \mathrm{o}, \mathrm{g}$,

$$
\begin{aligned}
\underline{\mathbf{X}} & =\left[\underline{X}_{i j}\right] \\
& =\left[\frac{d \underline{S}_{i}}{d p_{j}}\right] \\
& =\frac{1}{\operatorname{det} \mathbf{A}}\left[\begin{array}{ccc}
-A_{22} & A_{12}+A_{22} & -A_{12} \\
A_{21} & -\left(A_{11}+A_{21}\right) & A_{11}
\end{array}\right] .
\end{aligned}
$$




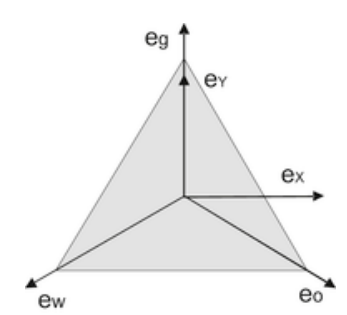

Fig. A.1. Axes $\left(\mathbf{e}_{X}, \mathbf{e}_{Y}\right)$ in the plane of the triangle $\mathscr{T}$.

The $2 \times 2$ matrix $d \underline{\mathbf{S}} / d \mathbf{S}$ is obtained by expressing the effective saturations in the axes $(X, Y)$ shown on Fig. A1, say componentwise,

$\frac{d \underline{S}_{\mathrm{w}}}{d S_{\mathrm{w}}}=\frac{1}{2 \sqrt{2} T_{r}}\left(\sqrt{3}(K J)_{X}-(K J)_{Y}\right) ; \quad \frac{d \underline{S}_{\mathrm{w}}}{d S_{\mathrm{o}}}=\frac{1}{2 \sqrt{2} T_{r}}(\sqrt{3}(K J)$. $\frac{d \underline{S}_{0}}{d S_{\mathrm{w}}}=\frac{1}{2 \sqrt{2} T_{r}}\left(\sqrt{3}(I K)_{X}-(I K)_{Y}\right) ; \quad \frac{d \underline{S}_{0}}{d S_{\mathrm{o}}}=\frac{1}{2 \sqrt{2} T_{r}}\left(\sqrt{3}(I K)_{i}\right.$

with $T_{r}$ the area of the irreducible triangle with vertices IJK shown on Fig. 1. The $2 \times 2$ matrix $d \mathbf{S} / d \underline{\mathbf{S}}$ required in (A.6) is deduced by inversion and next extended to a $3 \times 2$ matrix incorporating the gas saturation. Henceforth the derivatives of the saturations,

$\overbrace{\mathbf{X}}^{3 \times 3}=\left[X_{i j}\right]=\left[\frac{d S_{i}}{d p_{j}}\right]=\frac{d \mathbf{S}}{d \mathbf{p}}=\overbrace{\frac{d \underline{\mathbf{S}}}{d \underline{\mathbf{S}}}}^{3 \times 2} \overbrace{\underline{\mathbf{X}}}^{2 \times 3}$.

The derivatives satisfy the constraints,

$$
\begin{aligned}
\sum_{j=1,3} \frac{\partial S_{j}}{\partial p_{k}} & =0, \quad k \\
& \in[1,3], \quad \sum_{k=1,3} \frac{\partial S_{j}}{\partial p_{k}} \\
& =0, \quad j \\
& \in[1,3],
\end{aligned}
$$

and the same relations hold for the effective saturations,

$$
\begin{aligned}
\sum_{j=1,3} \frac{\partial \underline{S}_{j}}{\partial p_{k}} & =0, \quad k \\
& \in[1,3], \quad \sum_{k=1,3} \frac{\partial \underline{S}_{j}}{\partial p_{k}} \\
& =0, \quad j \\
& \in[1,3],
\end{aligned}
$$

\section{A.2. Capillary pressure matrix}

For the capillary pressures, expressed in eqn (5), the coefficients of matrix $\mathbf{A}$ in (A.2) are identified from the differentials,

$$
\begin{array}{r}
d p_{\mathrm{o}}-d p_{\mathrm{w}}=\left[30 f\left(1-\underline{S}_{\mathrm{o}}\right) \underline{S}_{\mathrm{o}}+1 c\right. \\
+\left[-30 f^{\prime}\left(1-\underline{S}_{\mathrm{o}}\right) c+30 f\left(1-\underline{S}_{\mathrm{o}}\right)\left(\underline{S}_{\mathrm{o}}\right.\right. \\
d p_{\mathrm{g}}-d p_{\mathrm{o}}=\left[40 f^{\prime}\left(1-\underline{S}_{\mathrm{g}}\right) a-40 f\left(1-\underline{S}_{\mathrm{g}}\right) \times 0.025 \times(1\right. \\
+\left[40 f^{\prime}\left(1-\underline{S}_{\mathrm{g}}\right) a-40 f\left(1-\underline{S}_{\mathrm{g}}\right)+30 f^{\prime}\left(1-\underline{S}_{\mathrm{o}}\right) b\right.
\end{array}
$$

where $f$ is the van Genuchten function defined by eqn. (4). and $f^{\prime}(x)$ denotes the derivative of $f$ with respect to its argument, i.e. $d f=f^{\prime} d x$,

$$
\begin{aligned}
f(\underline{S}) & =\left(\underline{S}^{\frac{-1}{m}}-1\right)^{\frac{1}{n}}, \quad f^{\prime} \\
& =\frac{d f}{d \underline{S}} \\
& =-\frac{1}{m n}\left(\underline{S}^{\frac{-1}{m}}-1\right)^{-m} \underline{S}^{\frac{-1}{m}-1}, \quad f^{\prime \prime} \\
& =\frac{1}{m n}\left(\underline{S}^{\frac{-1}{m}}-1\right)^{-m-1} \underline{S}^{\frac{-2}{m}-2}\left(\frac{1}{m}\right. \\
& \left.-\left(1+\frac{1}{m}\right) \underline{S}^{\frac{1}{m}}\right) .
\end{aligned}
$$

\section{A.3. Calculation of the derivatives $\partial D_{k} / \partial p_{j}$}

To calculate the derivatives $\partial D_{k} / \partial p_{j}, k, j=\mathrm{w}, \mathrm{o}, \mathrm{g}$ needed in eqn (27), the diffusion coefficient is defined by eqn. (15),

$D_{k}=\rho_{k} k_{r k} \frac{k_{\text {in }}}{\eta_{k}}, \quad \frac{1}{D_{k}} \frac{\partial D_{k}}{\partial p_{j}}=\frac{1}{K_{k}} I_{k j}+\frac{1}{k_{r k}} \frac{\partial k_{r k}}{\partial p_{j}}$,

where the bulk moduli of water and oil are constant while $K_{\mathrm{g}}=p_{\mathrm{g}}$. The viscosities are considered independent of the pressures. The relative permeabilities for a three-fluid phase mixture based on the Brooks-Corey model take the explicit form, namely, for a water wet system,

$$
\begin{aligned}
k_{r \mathrm{w}}= & \underline{S}_{\mathrm{w}}^{2 / \lambda+3}, \quad k_{r \mathrm{o}} \\
= & \underline{S}_{\mathrm{o}}^{2 / \lambda+3} \\
& +\underline{S}_{\mathrm{w}} \underline{S}_{\mathrm{o}}^{2}\left(\frac{1-\underline{S}_{\mathrm{o}}^{2 / \lambda+1}}{1-\underline{S}_{\mathrm{o}}}-\left(1-\underline{S}_{\mathrm{o}}\right)^{2 / \lambda}\right), \quad k_{r \mathrm{~g}} \\
= & \underline{S}_{\mathrm{g}}^{2}\left(1-\left(1-\underline{S}_{\mathrm{g}}\right)^{2 / \lambda+1}\right) .
\end{aligned}
$$

Hence, for $j=\mathrm{w}, \mathrm{o}, \mathrm{g}$,

$$
\begin{aligned}
\frac{1}{k_{r \mathrm{w}}} \frac{\partial k_{r \mathrm{w}}}{\partial p_{j}} & =\left(\frac{2}{\lambda}+3\right) \frac{1}{\underline{S}_{\mathrm{w}}} \frac{\partial \underline{S}_{\mathrm{w}}}{\partial p_{j}} ; \frac{\partial k_{r \mathrm{w}}}{\partial p_{j}} \\
& =\left(\frac{2}{\lambda}+3\right) \underline{S}_{\mathrm{w}}^{2 / \lambda+2} \frac{\partial \underline{S}_{\mathrm{w}}}{\partial p_{j}} ;
\end{aligned}
$$




$$
\begin{array}{r}
\frac{\partial k_{r \mathrm{o}}}{\partial p_{j}}=\underline{S}_{\mathrm{o}}^{2}\left(\frac{1-\underline{S}_{\mathrm{o}}^{2}}{1-\underline{\underline{i}}^{\prime}}\right. \\
+\left[\left(\frac{2}{\lambda}+3\right) \underline{S}_{\mathrm{o}}^{2 / \lambda+2}+\frac{\underline{S}_{\mathrm{w}} \underline{S}_{\mathrm{o}}}{\left(1-\underline{S}_{\mathrm{o}}\right)^{2}} \times\left(2-\underline{S}_{\mathrm{o}}+(2 / \lambda+2) \underline{S}_{\mathrm{o}}^{2 / \lambda+2}\right.\right. \\
\frac{1}{k_{r \mathrm{~g}}} \frac{\partial k_{r \mathrm{~g}}}{\partial p_{j}}=\left(\frac{2}{\underline{S}_{\mathrm{g}}}+\left(\frac{2}{\lambda}+1\right) \frac{\left(1-\underline{S}_{\mathrm{g}}\right)^{2 / \lambda}}{\left.1-\left(1-\underline{S}_{\mathrm{g}}\right)^{2 / \lambda+1}\right)} \frac{\partial \underline{S}_{\mathrm{g}}}{\partial p_{j}}\right. \\
\left(2 \underline{S}_{\mathrm{g}}\left(1-\left(1-\underline{S}_{\mathrm{g}}\right)^{2 / \lambda+1}\right)+\left(\frac{2}{\lambda}+1\right) \underline{S}_{\mathrm{g}}^{2}\left(1-\underline{S}_{\mathrm{g}}\right)\right.
\end{array}
$$

the derivatives $d \underline{S}_{i} / d p_{j}$ being given by (A.4).

\section{References}

Al-Dhahli, A., van Dijke, M.I.J., Geiger, S., 2013. Accurate modelling of pore-scale films and layers flow for three-phase flow processes in clastic and carbonate rocks with arbitrary wettability. Transport Porous Media 98, 259-286.

Amiri, S.G., Sadrnejad, S.A., Ghasemzadeh, H., Montazeri, G.H., 2013. Application of control volume based finite element method for solving the black-oil fluid equations. Petrol. Sci. 10 (3), 361-372.

Bass, D.H., Hastings, N.A., Brown, R.A., 2000. Performance of air sparging systems: a review of case studies. J. Hazard Mater. 72 (2), 101-119.

Bear, J., Verruijt, A., 1987. Modeling Groundwater Flow and Pollution. D. Reidel, Dordrecht, Holland.

Behbahani, H., Blunt, M.J., 2005. Analysis of imbibition in mixed-wet rocks using pore-scale modeling. Soc. Petrol. Eng. J. 466-473.

Bell, J.B., Trangenstein, J.A., Shubin, G.R., 1986. Conservation laws of mixed type describing three-phase flow in porous media. SIAM J. Appl. Math. 46 (6), 1000-1017.

Binning, P., Celia, M.A., 1999. Practical implementation of the fractional flow approach to multi-phase flow simulation. Adv. Water Resour. 22 (5), 461-478.

Bowen, R.M., 1976. Theory of mixtures. In: In: Eringen, A.C. (Ed.), Continuum Physics, vol. III, Academic Press, New York, USA.

Butler, R.M., 1994. Steam-assisted gravity drainage: concept, development, performance and future. J. Can. Petrol. Technol. 33 (2), 44-50.

Casulli, V., Zanolli, P., 2010. A nested Newton-type algorithm for finite volume methods solving Richards' equation in mixed form. SIAM J. Sci. Comput. 32 (4), 2255-2273.

Celia, M.A., Bouloutas, E.T., Zarba, R.L., 1990. A general mass-conservative numerical solution for the unsaturated flow equation. Water Resour. Res. 26 (7), 1483-1496.

Chavent, G., Jaffré, J., 1978. Mathematical Models and Finite Elements for Reservoir Simulation. North-Holland, Amsterdam, The Netherlands.

Chen, Z., 2000. Formulations and numerical methods of the black oil model in porous media. SIAM J. Numer. Anal. 38 (2), 489-514.

Chen, Z., Ewing, R.E., 1997. Comparison of various formulations of three-phase flow in porous media. J. Comput. Phys. 132 (2), 362-373.

Egermann, P., Mejdoub, K., Lombard, J.M., Vizika, O., Kalam, Z., 2013. Drainage three-phase flow relative permeability on oil-wet carbonate reservoir rock-types: experiments, interpretation and comparison with standard correlations. International Symposium of the Society of Core Analysts 14, Napa Valley, 16-19 September 2013, Paper Number SCA2013-A028.

El-Amin, M.F., Sun, S., 2011. Effects of gravity and inlet/outlet location on two-phase cocurrent imbibition in porous media. J. Appl. Math. 18. https://doi.org/10.1155/ 2011/673523, Article ID 673523.
Faust, C.R., 1985. Transport of immiscible fluids within and below the unsaturated zone: a numerical model. Water Resour. Res. 21 (4), 587-596.

Forsyth, P.A., 1994. Three dimensional modelling of steam flush for DNAPL site remediation. Int. J. Numer. Meth. Fluid. 19 (12), 1055-1081.

Forsyth, P.A., Shao, B.Y., 1991. Numerical simulation of gas venting for NAPL site remediation. Adv. Water Resour. 14 (6), 354-367.

Gajo, A., Cecinato, F., Loret, B., 2017. Deformable porous media saturated by three immiscible fluids: constitutive modelling and simulations of injection and imbibition tests. Transport Porous Media 116 (1), 19-51.

Harari, I., 2004. Stability of semidiscrete formulations for parabolic problems at small time steps. Comput. Meth. Appl. Mech. Eng. 193 (15), 1491-1516.

Harari, I., Hauke, G., 2007. Semidiscrete formulations for transient transport at small time steps. Int. J. Numer. Meth. Fluid. 54 (6-8), 731-743.

Hauke, G., Doweidar, M.H., 2004. Exploring new subgrid scale stabilized methods for advection-diffusion- reaction. In: Neittaanmaki, P., et al. (Eds.), Proc. ECCOMAS 2004.

Hustad, O.S., March 2000. Simulation Examples Using a Fully Coupled Model for Three-phase Capillary Pressure and Relative Permeability with Hysteresis. Sintef Petroleum Research, Trondheim, Norway.

Hustad, O.S., 2002. A coupled model for three-phase capillary pressure and relative permeability. Soc. Petrol. Eng. J. 7 (1), 59-69.

Juanes, R., Patzek, T., 2004. Relative permeabilities for strictly hyperbolic models of three-phase flow in porous media. Transport Porous Media 57, 125-152.

Khalili, N., Khabbaz, M.H., 1995. On the theory of three-dimensional consolidation in unsaturated soils. In: Proc. Of the First International Conference on Unsaturated Soils, Paris, France.

Leverett, M.C., Lewis, W.B., 1941. Steady flow of gas-oil-water mixtures through unconsolidated sands. Petroleum Transactions of the AIME 142 (1), 107-116.

Loret, B., Khalili, N., 2000. A three phase model for unsaturated soils. Int. J. Numer. Anal. Meth. GeoMech. 24 (11), 893-927.

Ma, S., Morrow, N.R., Zhang, X., 1997. Generalized scaling of spontaneous imbibition data for strongly water-wet systems. J. Petroleum Engineering and Science 18, 165-178.

Mercer, J.W., Cohen, R.M., 1990. A review of immiscible fluids in the subsurface: properties, models, characterization and remediation. J. Contam. Hydrol. 6 (2), 107-163.

Mercer, J.W., Faust, C.R., 1976. The application of finite-element techniques to immiscible flow in porous media. In: Proc. 1st Int. Conf. On Finite Elements in Water Resources. Princeton University, USA.

Morel-Seytoux, H.J., 1973. Two-phase flows in porous media. In: In: Chow, V.T. (Ed.), Advances in Hydroscience, vol. 9, Academic Press, New York, pp. 119-202.

Mosharaf Dehkordi, M.T., Manzari, M., Ghafouri, H., Fatehi, R., 2014. A general finite volume based numerical algorithm for hydrocarbon reservoir simulation using blackoil model. Int. J. Numer. Meth. Heat Fluid Flow 24 (8), 1831-1863.

Mroginski, J.L., Di Rado, H.A., Beneyto, P.A., Awruch, A.M., 2010. A finite element approach for multiphase fluid flow in porous media. Math. Comput. Simulat. 81 (1), 76-91.

Neuman, S.P., 1973. Saturated-unsaturated seepage by finite elements. ASCE J. Hydraulics Division 2233-2250, (HY12) ed.

Pao, W.K., Lewis, R.W., Masters, I., 2001. A fully coupled hydro-thermo-poro-mechanical model for black oil reservoir simulation. Int. J. Numer. Anal. Meth. GeoMech. 25 (12), 1229-1256.

Peaceman, D.W., 1977. Fundamentals of Numerical Reservoir Simulation. Elsevier, New York.

Plug, W.J., Mazumder, S., Bruining, J., 2008. Capillary pressure and wettability behavior of $\mathrm{CO}^{2}$ sequestration in coal at elevated pressures. Soc. Petrol. Eng. J. 455-464.

Spivak, A., Price, H.S., Settari, A., 1977. Solution of the equations for multidimensional, two-phase, immiscible flow by variational methods. In: Fourth SPE Symposium on Numerical Simulation of Reservoir Performance, Los Angeles, USA.

Yang, D., Moridis, G.J., Blasingame, T.A., 2014. A fully coupled multiphase flow and geomechanics solver for highly heterogeneous porous media. J. Comput. Appl. Math. 270, 417-432.

Yin, S., Dusseault, M.B., Rothenburg, L., 2009. Thermal reservoir modeling in petroleum geomechanics. Int. J. Numer. Anal. Meth. GeoMech. 33 (4), 449-485. 